\title{
Improving XCP to Achieve Max-Min Fair Bandwidth Allocation
}

\author{
Xiaowei Yang, Yanbin Lu, and Lei Zan \\ University of California, Irvine \\ $\{x w y$, yanbinl, lzan $\} @$ ics.uci.edu
}

\begin{abstract}
TCP is prone to be inefficient and unstable in high speed and long latency networks [1]. The eXplicit Control Protocol (XCP) is a new and promising protocol that outperforms TCP in terms of efficiency, stability, queue size, and convergence speed. However, Low et al. recently discovered a weakness of XCP. In a multi-bottleneck environment, XCP may achieve as low as $80 \%$ utilization at a bottleneck link and consequently some flows may only receive a small fraction of their max-min fair rates.

This paper proposes $\mathrm{iXCP}$, an improved version of XCP. Extensive simulations show that $\mathrm{iXCP}$ overcomes the weakness of XCP, and achieves efficient and fair bandwidth utilization in both single- and multi- bottleneck environments. In addition, we prove that iXCP is max-min fair in steady state. This result implies that iXCP is able to fully utilize bottleneck bandwidth. Simulations show that iXCP preserves the good properties of XCP, including negligible queue lengths, near-zero packet loss rates, scalability, and fast convergence. Simulations also show that iXCP overcomes the under-utilization and instability problem of P-XCP [2], and outperforms JetMax [3] in terms of link utilization in the presence of highly dynamic traffic.
\end{abstract}

\section{INTRODUCTION}

It is well known that TCP's congestion control mechanism is inefficient and instable in high bandwidth-delay-product environments [4]-[7]. TCP treats a packet loss as an implicit congestion signal, and reacts to congestion by cutting its congestion window size by half, and then gradually increases its window size by one every round trip time (RTT). This sawtoothed behavior of TCP leads to throughput oscillation and link under-utilization, especially in a high bandwidth-delayproduct environment.

The eXplicit Control Protocol (XCP) [8] overcomes the limitations of TCP by sending explicit window adjustment information from routers to end hosts. It is a promising candidate to replace TCP in a future Internet architecture [9]. Unlike TCP, an XCP flow does not implicitly probe available bandwidth. Instead, a router computes the spare bandwidth of an output link, and fairly allocates the bandwidth among all flows that share the same link. The router then writes the amount of window adjustment in the congestion header of an XCP flow. This explicit control mechanism allows a flow to quickly utilize the available bandwidth of a link. Early results have shown that XCP is highly efficient, stable, and scalable [8].

However, Low et al. [10] recently revealed a weakness of XCP. In a multi-bottleneck environment, XCP's congestion control mechanism may cause some bottleneck link to be under-utilized, and a flow may only receive a small fraction of its max-min fair allocation [11]. ${ }^{1}$ Low et al. demonstrated this result with both a theoretical model and simulations. With the chosen parameters, XCP may utilize only $80 \%$ of bottleneck bandwidth in the worst case. In this paper, we refer to this theoretical model as Low's model.

Intuitively, this problem occurs because XCP-enabled routers independently compute bandwidth allocation. For instance, if a flow is bottlenecked at a downstream link, an upstream router would still attempt to allocate bandwidth to that flow to ensure local fairness. This leads to link underutilization, which in turn causes some flow to receive only a fraction of its max-min fair allocation.

In this paper, we propose a simple improvement to XCP that overcomes this limitation. We add an additional bottleneck identifier field into XCP's congestion header. If a flow is bottlenecked at an outgoing link of a router, the router writes the link identifier into this field. Other routers are then aware that the flow is not bottlenecked at their links. We further modify XCP's control algorithm not to waste bandwidth on flows that are bottlenecked at other routers.

We use extensive simulations to show that our improved $\mathrm{XCP}$ (iXCP) is able to achieve nearly $100 \%$ link utilization and max-min fairness in steady state. We use a theoretical model to show that $\mathrm{iXCP}$ is max-min fair. This result also implies that iXCP is able to fully utilize bottleneck bandwidth. In addition, our simulation results show that $\mathrm{iXCP}$ preserves the desirable features of XCP. It converges fast to max-min bandwidth allocation with a negligible queue length; it is efficient and fair in high speed and long latency networks as well as conventional networks; it also remains as a scalable stateless solution. Simulations also show that $\mathrm{iXCP}$ overcomes the under-utilization and instability problem of P-XCP [2], and outperforms JetMax [3] in terms of link utilization in the presence of highly dynamic traffic.

This paper has three key contributions. The first is our analysis on the root cause of XCP's under-utilization problem. Although this problem has been observed in [2], [10], to the best of our knowledge, we are the first to pinpoint the particular protocol mechanism that causes the problem. The

\footnotetext{
${ }^{1} \mathrm{~A}$ max-min fair allocation maximizes the bandwidth allocated to the flow with a minimum allocation. Max-min fairness satisfies the following conditions: 1) the allocation is feasible, i.e., the sum of the allocated rates does not exceed a link's capacity; 2) a flow's rate allocation cannot be increased without violating the feasibility constraint or without decreasing the rate of some flow that has an equal or smaller bandwidth share.
} 
second is our improvement to XCP. This improvement makes $\mathrm{XCP}$ achieve its full potential: $\mathrm{XXCP}$ is highly efficient and fair in all types of topologies. The third is the theoretical analysis that shows iXCP is max-min fair. This analysis provides us the confidence that $\mathrm{iXCP}$ will continue to operate efficiently and fairly in scenarios that we did not simulate. We note that our theoretical analysis builds on Low's model [10].

The rest of the paper is organized as follows. In section II, we briefly summarize how XCP works and its weakness. Section III describes iXCP. We use extensive simulations to evaluate the performance of iXCP in Section IV. Appendix presents our theoretical analysis that proves iXCP is max-min fair and stable. We compare our work with related work in section V. Section VI concludes the paper.

\section{UNDERSTANDING THE WEAKNESS OF XCP}

Understanding what causes a problem is the first step to solve the problem. In this section, we describe how XCP works and analyze what makes XCP under-utilize link bandwidth.

\section{A. How XCP works}

$\mathrm{XCP}$ uses explicit window adjustment for a flow to increase or decrease its congestion window. Each packet carries a congestion header that contains three fields: the sender's current congestion window size: $c w n d$, the estimated round trip time: $r t$, and the router feedback field: feedback. Each sender fills its current cwnd and rtt values in the congestion header on packet departures, and initializes the feedback field to its desired window size.

The core control mechanism of XCP is implemented at routers. Each router has two logical controllers: efficiency controller and fairness controller. In each control interval, the efficiency controller computes spare bandwidth as follows:

$$
\phi=\alpha d(c-y)-\beta b
$$

where $d$ is the average round-trip time, $c$ is the link capacity, $y$ is the input traffic rate, and $b$ is the persistent queue length. $\alpha$ and $\beta$ are two control parameters, with default value 0.4 and 0.226 respectively.

The fairness controller is responsible for fairly allocating bandwidth to each flow. When a link is in high-utilization region, XCP's fairness controller performs a "bandwidth shuffling" operation to ensure local fairness. This operation simultaneously allocates and deallocates the shuffled bandwidth among flows. The shuffled bandwidth is computed as follows:

$$
h=\max \{0, \gamma y-|\phi|\}
$$

where $\gamma$ is a control parameter with default value $10 \%$.

In each control interval, $h+\max (\phi, 0)$ are allocated to each flow additively, i.e., each flow gets an equal amount of increment. At the mean time, $h+\max (-\phi, 0)$ is deallocated multiplicatively, i.e., each flow gets a rate decrement proportional to its current rate. A router writes the net window adjustment (the increment minus the decrement) information in the feedback field of a packet. A more congested downstream router may overwrite this field with a smaller increment or a larger decrement. The receiver echoes back the feedback to the sender, and the sender adjusts its window size accordingly.

\section{B. The Weakness Revealed}

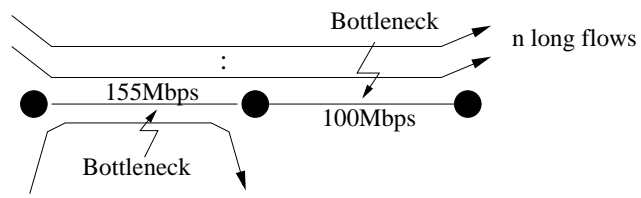

Fig. 1. In this topology, XCP under-utilizes the $155 \mathrm{Mb} / \mathrm{s}$ link. The $n$ long flows are bottlenecked at the $100 \mathrm{Mb} / \mathrm{s}$ link. So the short flow should be able to send at $55 \mathrm{Mb} / \mathrm{s}$, but with XCP, it may get a bandwidth allocation anywhere between $24 \mathrm{Mb} / \mathrm{s}$ to $55 \mathrm{Mb} / \mathrm{s}$, depending on the number of long flows.

Low et al. rigorously modeled XCP's control algorithm in [10]. Both their analysis and simulations revealed that XCP may under-utilize a bottleneck link. In the worst case, XCP may only achieve $80 \%$ link utilization (with XCP's default parameters). We describe this problem using the network topology shown in Figure 1. This is an example used by Low et al. in [10]. In this example, there are $n(n \geq 2)$ long flows that cross both links in the figure and a short flow that only goes through the $155 \mathrm{Mb} / \mathrm{s}$ link. Since each long flow is bottlenecked at the $100 \mathrm{Mb} / \mathrm{s}$ link, in a max-min fair allocation, each of them gets a bandwidth allocation of $100 / n \mathrm{Mb} / \mathrm{s}$. The short flow should fully utilize the residual bandwidth of the $155 \mathrm{Mb} / \mathrm{s}$ link, obtaining a $55 \mathrm{Mb} / \mathrm{s}$ bandwidth allocation. However, both simulations and analysis show that $\mathrm{XCP}$ allocates a rate $r(n)<55 \mathrm{Mb} / \mathrm{s}$ bandwidth to the short flow in steady state. The function $r(n)$ decreases as $n$ increases. Figure 4(a) and 4(b) show the utilization of the $155 \mathrm{Mb} / \mathrm{s}$ bottleneck link and the ratio between the short flow's allocated rate and its max-min fair share respectively. As we can see, both the link utilization and the short flow's bandwidth allocation decrease as the number of long flows $n$ increases.

Intuitively, this problem occurs because XCP-enabled routers independently allocate bandwidth to each flow based on their local information. Although a long flow is bottlenecked at the downstream $100 \mathrm{Mb} / \mathrm{s}$ link, the upstream router at the $155 \mathrm{Mb} / \mathrm{s}$ link still attempts to increase its bandwidth share to ensure local fairness. As a result, the short flow that is only bottlenecked at the upstream link cannot fully utilize the link and obtain its max-min fair share.

We explain it in more depth to shed light on the solution. The problem is primarily caused by XCP's fairness controller. The "bandwidth shuffling" operation essentially uses the Additive-Increase Multiplicative-Decrease (AIMD) algorithm to adjust rates among flows. Thus, a flow with a larger bandwidth share will be tariffed more and get back less, and vice versa. In a single bottleneck environment, the $A I M D$ policy will equalize all flow's bandwidth shares, thereby achieving fairness.

The problem arises in a multi-bottleneck environment. In such an environment, the de-allocated bandwidth from a shuffling operation may not be fully utilized, when some flows are bottlenecked at other links (no matter downstream or upstream links) and cannot further increase their sending rates. This deallocated but not re-used bandwidth leads to link under-utilization. The flows that could have used this bandwidth have a net loss in a shuffling operation. Recall that 
the fairness controller is also in charge of allocating the spare bandwidth computed by the efficiency controller. When the net loss of a flow from the shuffling operation balances out its net gain in the spare bandwidth allocation, the system has reached an equilibrium, in which a flow's sending rate cannot further increase although there remains un-used bandwidth.

We illustrate this problem with a numerical example. In the example shown in Figure 1, when there are four long flows, with $\mathrm{XCP}$, the equilibrium rate allocation for the short flow is $43 \mathrm{Mb} / \mathrm{s}$, and the rate for each long flow is $25 \mathrm{Mb} / \mathrm{s}$. This leads to a $12 \mathrm{Mb} / \mathrm{s}$ under-utilization at the $155 \mathrm{Mb} / \mathrm{s}$ link. We show that this is an equilibrium using XCP's default parameters [8]. At the $155 \mathrm{Mb} / \mathrm{s}$ upstream link, the used bandwidth is $143 \mathrm{Mb} / \mathrm{s}$ and the unused bandwidth is $12 \mathrm{Mb} / \mathrm{s}$. The efficiency controller will compute the spare bandwidth as $0.4 * 12=4.8 \mathrm{Mb} / \mathrm{s}$. The fairness controller will compute the shuffled bandwidth as $0.1 * 143-4.8=9.5 \mathrm{Mb} / \mathrm{s}$. The additive increase for each flow is the sum of the spare and shuffled bandwidth evenly distributed among all flows: $(4.8+9.5) / 5=2.86 \mathrm{Mb} / \mathrm{s}$; the multiplicative decrease for the short flow is proportional to its rate: $9.5 / 143 * 43=2.86 \mathrm{Mb} / \mathrm{s}$, and similarly, the decrease for a long flow is $9.5 / 143 * 25=1.66 \mathrm{Mb} / \mathrm{s}$. The decrease for the short flow is the same as the increase for the short flow, and the long flows cannot increase beyond $25 \mathrm{Mb} / \mathrm{s}$ due to the $100 \mathrm{Mb} / \mathrm{s}$ bottleneck link. Therefore, no flow's rate can change over time. The system has reached an equilibrium, in which $12 \mathrm{Mb} / \mathrm{s}$ bandwidth is not used.

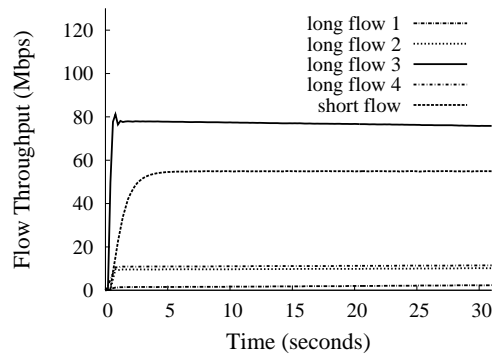

Fig. 2. When there is no shuffling operation, XCP can achieve full link utilization but no fairness ensured.

One might think that if the problem is caused by the bandwidth shuffling operation, we can simply disable bandwidth shuffling to fix the problem. However, this simple fix does not work. Bandwidth shuffling is essential to prevent starvation for new flows and to achieve some degree of fairness. The efficiency controller of XCP will not make any rate adjustment when the bandwidth is fully utilized. Without bandwidth shuffling, XCP can achieve a full link utilization, but the rate allocations to different flows can be arbitrarily unfair. When existing flows have used up a link's bandwidth, a new flow may be starved. As an example, if we disable bandwidth shuffling in Figure 1, four long flows and a short flow may obtain a bandwidth allocation as shown in Figure 2. Three long flows that start late are almost starved.

\section{IMPROVED XCP (IXCP)}

In this section, we describe our improvement to XCP. As we discussed in the previous section, the under-utilization problem

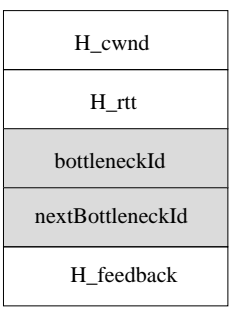

Fig. 3. Congestion header for iXCP. We add two additional fields: the bottleneckId field and the nextBottleneckId field.

is caused by a router's attempt to shuffle bandwidth from flows that can further increase their rates to flows that cannot, i.e., flows that are bottlenecked at other routers. Therefore, we modify XCP's control algorithm to shuffle bandwidth only among flows that are bottlenecked at current routers. This modification ensures that the deallocated bandwidth by the shuffling operation will be re-used. Therefore, a link will be fully utilized.

To implement this improvement, a router must be aware whether a flow is bottlenecked at the router or not. For a router to obtain this information, we include two additional fields in the XCP's congestion header: the bottleneckId and the nextBottleneckId field, as shown in Figure 3. Each router uses bottleneckId to identify the flows that are bottlenecked at this router and computes the traffic to be shuffled based on bottlenecked flows. A router sets the nextBottleneckId field on the fly based on the feedback value. If the feedback from this router is non-positive and is smaller than the one in the packet header, then the outgoing link of this router becomes the new bottleneck for the flow, and the router writes its outgoing link identifier to this field. Note that a router does not modify the nextBottleneckId field as long as its feedback is positive. This is because if a flow receives a positive feedback at a router, it indicates that the flow has not reached its fair share of bandwidth at the router yet and it does not know whether it will be bottlenecked at this router or not. Therefore the router need not change the flow's bottleneckId field. To ensure uniqueness, a router may use the IP address of an interface card or a random 32-bit value as a link identifier. Initially, both the bottleneckId and the nextBottleneckId fields are set to a sender's access link identifier. An iXCP receiver acknowledges back the feedback and the nextBottleneckId field to the sender. The sender copies the nextBottleneckId field from the acknowledgement to the bottleneckId field of its outgoing packets.

The pseudo-code of the algorithm for routers is presented in Algorithm 1. The control algorithm of XCP is modified as follows. On a packet arrival, a router estimates the spare bandwidth of an outgoing link as in the original XCP, and the router estimates the shuffled bandwidth only from those packets whose bottleneckIds match the link identifier of the router (see lines 6-9 in Algorithm 1). The feedback computation is the same as in XCP. If the feedback from the router is non-positive and is smaller than the one in the packet header, then the router changes nextBottleneckId field (see line 31 in Algorithm 1).

Careful readers may note that we do not distinguish between bottlenecked flows and non-bottlenecked flows when generat- 


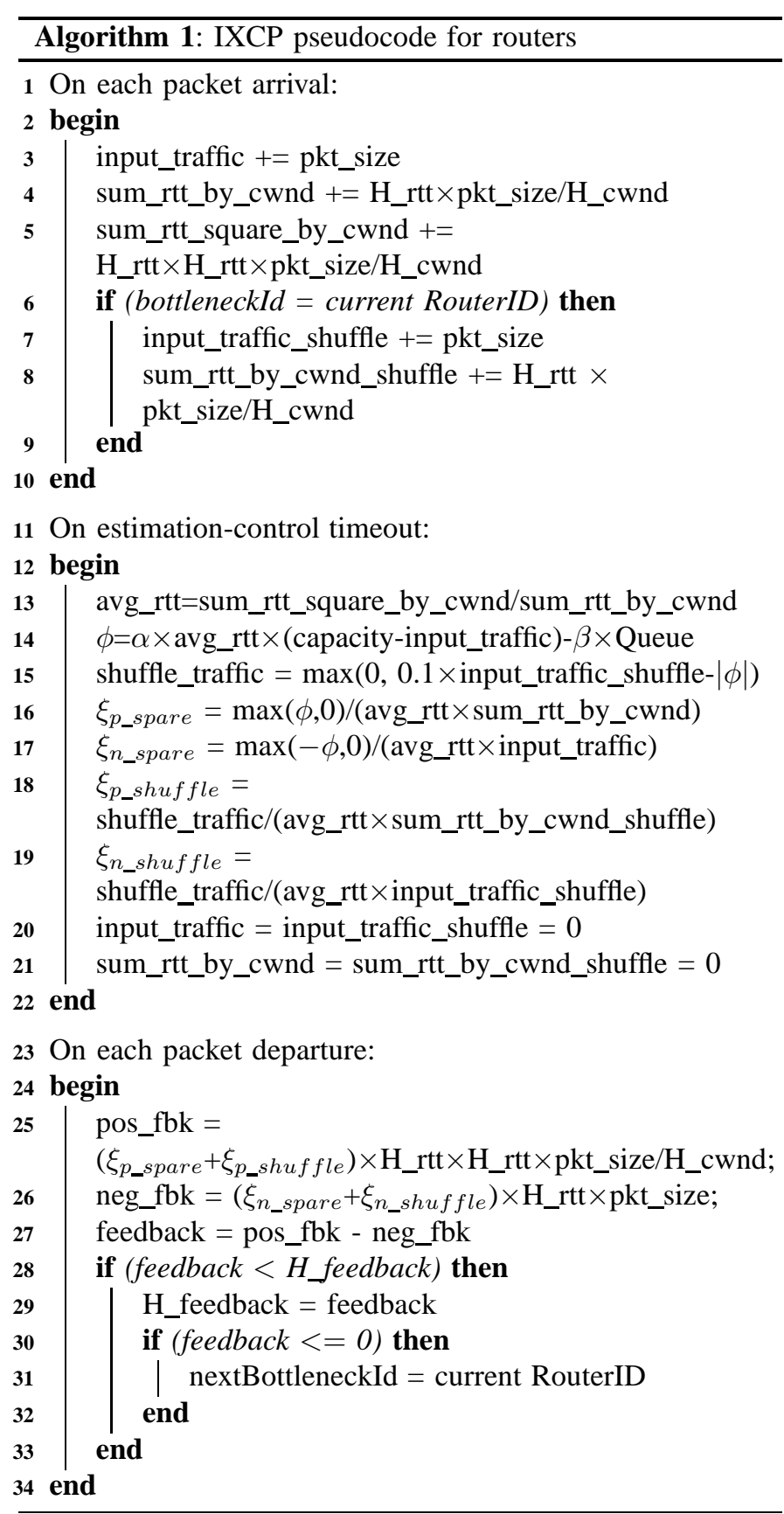

ing the feedback and that the shuffling factor, $\xi_{p_{-} s h u f f l e}$ and $\xi_{n \_s h u f f l e}$, computed based on bottlenecked flows may not be applied to non-bottlenecked flows. However, we showed, in Theorem B, that the bottleneckId of each flow will converge. We refer interesting readers to ns- 2 implementation of iXCP [12].

The drawback of our modification is that iXCP increases the congestion header of XCP by eight bytes and the acknowledgement header by four bytes. If we assume the average packet size is 400 bytes [13], and each packet has both a congestion header and an acknowledgement header, we increase the packet header overhead by $3 \%$. However, as we will show in the following sections, iXCP can increase link utilization by almost $20 \%$ in some multi-bottleneck environments. Besides, packet sizes may increase in the future due to advanced

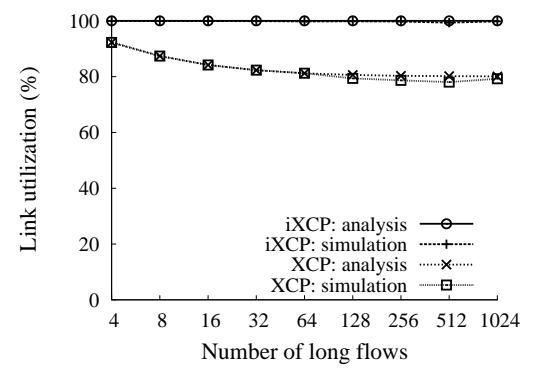

(a) Utilization for the $155 \mathrm{Mb} / \mathrm{s}$ link in Figure 1.

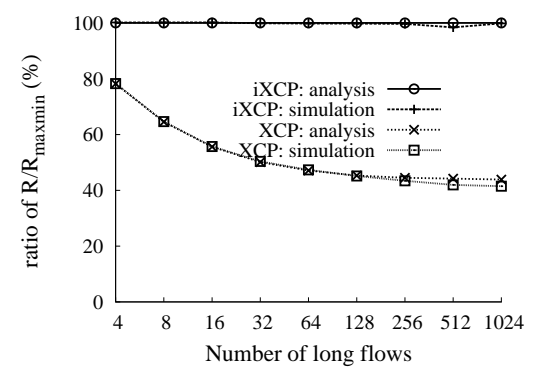

(b) Ratio of the short flow's rate $(R)$ over its max-min fair rate $\left(R_{\text {maxmin }}\right)$.

Fig. 4. iXCP achieves nearly optimal link utilization and maxmin fair rate for the short flow. The figures compare iXCP with $\mathrm{XCP}$ using both simulation and theoretical results.

technologies, e.g., gigabit Ethernet with a jumbo frame size of 9000 bytes. Therefore, we think it is a worthy tradeoff to design iXCP to achieve efficient and fair bandwidth allocation in both single- and multi-bottleneck topologies at the cost of slightly increased header overhead.

In Section A, we build on Low's theoretical model [10] to prove the following theorems:

Theorem A. iXCP achieves max-min fair bandwidth allocation in steady state. That is, with $i X C P$, every flow has a bottleneck. And a link is called bottleneck for a flow if and only if its sending rate is larger than or equal to all other flows passing through that link.

Theorem B. All flows' bottleneckIds converge to their correct bottleneck.

\section{Simulation Results}

In this section, we use ns2 [14] (version 2.3.2) simulations to evaluate the performance of $\mathrm{iXCP}$. The simulation code is available in [12].

The simulation scenarios include multiple bottleneck topologies, heterogeneous RTTs and web-like traffic. There are two key questions we aim to answer: 1) does iXCP fix the problem of XCP and achieve max-min fair allocation? 2) does iXCP make other properties of XCP worse? To answer the first question, we compute two metrics: 1) link utilization; 2) a flow's rate normalized by its theoretical max-min rate. If the link utilization is $100 \%$, and a flow's normalized rate is 1 , it shows that iXCP achieves max-min fair bandwidth allocation. To answer the second question, we examine three metrics: 1) 


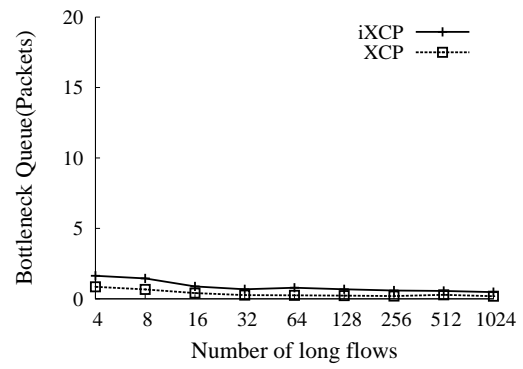

(a) Average queue size.

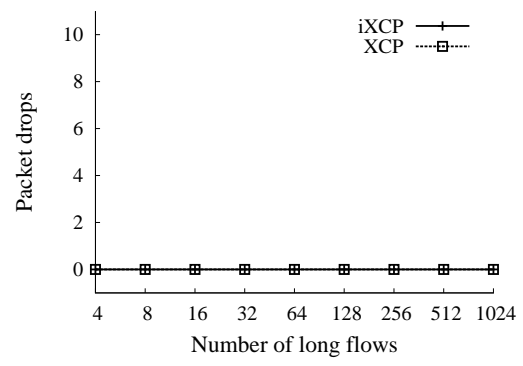

(b) Packet drops.

Fig. 5. Small average queue size and zero packet drops are achieved for the $155 \mathrm{Mb} / \mathrm{s}$ link in Figure 1.

average queue sizes; 2) the packet drops; 3) the convergence speed when flows join and leave the network.

In our simulations, we use the same parameter settings as those chosen by XCP [8] for the purpose of comparison. The coefficients for the efficiency controller $\alpha$ and $\beta$ are set to be 0.4 and 0.226 respectively, and the coefficient for the fairness controller $\gamma$ is set to be 0.1 . The packet size is 1000 bytes and the queue size is set to the delay-bandwidth product. The propagation delay of each link in all topologies is $20 \mathrm{~ms}$ unless otherwise noted, and the bandwidth of each link is specified in the figures.

\section{A. A simple two-bottleneck environment}

We simulated the scenario as shown in Figure 1. The short flow is bottlenecked at the first $155 \mathrm{Mb} / \mathrm{s}$ link, and all the other long flows are bottlenecked at the second $100 \mathrm{Mb} / \mathrm{s}$ link. We vary the number of long flows from 4 to 1024 . We only show the results for the short flow on the first link, because XCP cannot fully utilize the bandwidth of the first link and does not allocate the max-min rate to the short flow, as explained in Section II-B. The second link is fully utilized in both XCP and $\mathrm{iXCP}$, and each long flow obtains its max-min rate of $100 / n \mathrm{Mb} / \mathrm{s}$.

Figure 4(a) and 4(b) show the link utilization for the first bottleneck link and the ratio between the short flow's rate and its theoretical max-min share. We show both the simulation and the theoretical results for XCP and iXCP. Theoretical results for XCP are obtained using Low's model [10]; theoretical results for iXCP are obtained using our analysis described in Section A. As can be seen, as the number of long flows increases, the link utilization of XCP decreases and approaches its theoretical lower bound $80 \%$. The short flow only obtains $40 \%$ of its max-min rate. In contrast, iXCP achieves more than $99 \%$ link utilization; the short flow's rate is more than $98 \%$ of its max-min rate.

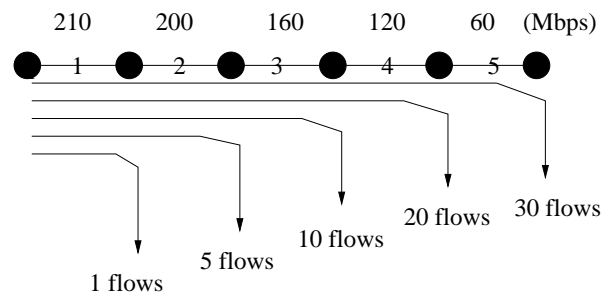

Fig. 6. A multi-bottleneck topology.

Figure 5(a) and 5(b) show the average queue size and the packet drops at the $155 \mathrm{Mb} / \mathrm{s}$ link. As can be seen, both XCP and $\mathrm{iXCP}$ have very small queue size, and negligible packet drops. (In the simulations, the packet drops are zero.)

\section{B. A multi-bottleneck environment}

We studied how iXCP performs in a multi-bottleneck environment as shown in Fig 6. In this topology, there are a total of five hops and all flows are bottlenecked at the last hop they cross. A link is labeled with an identifier ranging from 1 to 5 . For example, the thirty longest flows are bottlenecked at the fifth link; the twenty second longest flows are bottlenecked at the fourth link; and so on. The max-min rates for flows bottlenecked at link 1 to 5 are $10 \mathrm{Mbps}$, $8 \mathrm{Mbps}$, 4Mbps, $3 \mathrm{Mbps}$, 2Mbps, respectively.

Figure 7(a) and 7(b) show the utilization and the normalized flow rate at each link achieved by both iXCP and XCP. We only show the bottlenecked flows for each link, and the normalized rates are averaged over all bottlenecked flows. The standard deviations among flows are too small to be visible. Thus, they are not shown in the figure. As can be seen, iXCP achieves full link utilization and max-min rate allocations for all flows at all bottleneck links, while XCP cannot fully utilize the bandwidth of the first four links.

Figure 8(a) and 8(b) show the average queue size and packet drops for the multi-bottleneck topology. Both of them are small in XCP and iXCP.

\section{Dynamic flows}

We simulated the case in which the bottlenecks of flows change over time as flows dynamically join and leave the network. This simulation is to show how well iXCP converges when there are sudden traffic demand changes.

The simulation topology is shown in Figure 9. Each link has round-trip propagation delay $20 \mathrm{~ms}$. We start the long flow, and four short flows from $s_{1}$ to $s_{4}$ at time $t=0$. Each short flow only crosses one link. We then start three short flows $s_{5}, s_{6}$, and $s_{7}$ at $t=20$. At time $t=40$, the short flows from $s_{4}$ to $s_{7}$ stop.

Figure 10(b) shows the utilization of each link. As can be seen, $\mathrm{iXCP}$ is robust to the sudden changes in traffic demand. After four flows exist the system, there is only a temporary dip, and the link is quickly fully utilized again.

Figure 11(a), 11(b), and 11(c) show how each flow's rate changes over time as its bottleneck shifts with traffic demand in iXCP. All flows converge to their max-min rates shortly after a change in the network. In the first 20 seconds, the long flow is bottlenecked at the $120 \mathrm{Mb} / \mathrm{s}$ link. The max-min rates 


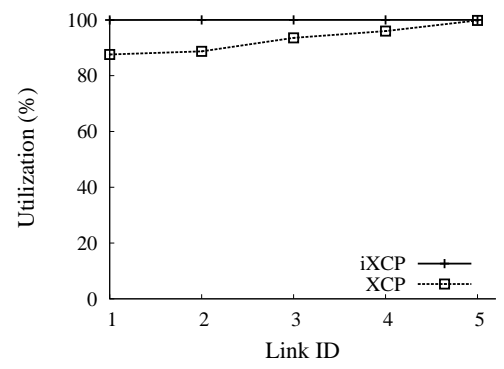

(a) Link utilization.

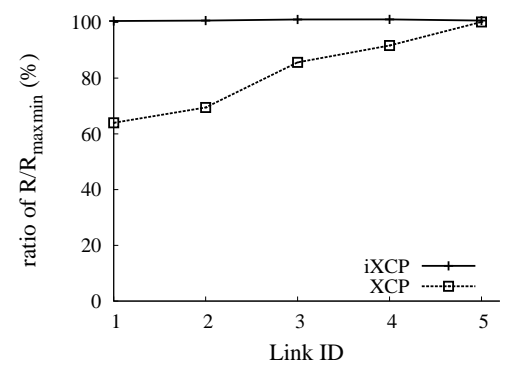

(b) Ratios of flow rates $(R)$ over their max-min fair rates $\left(R_{\text {maxmin }}\right)$.

Fig. 7. iXCP achieves nearly $100 \%$ link utilization and maxmin fair flow rates for each link in the multi-bottleneck topology shown in Figure 6.

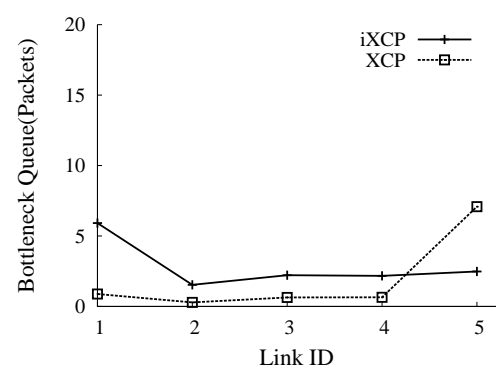

(a) Average queue size.

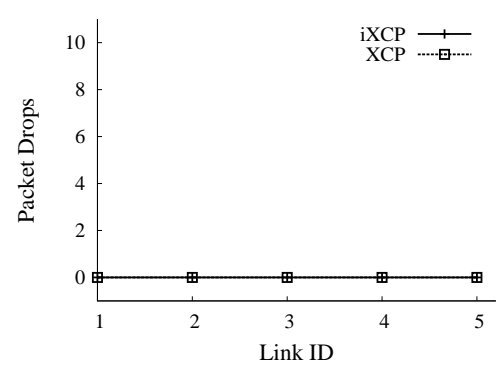

(b) Packet drops.

Fig. 8. iXCP achieves small average queue size and zero packet drops for each link in the multi-bottleneck topology shown in Figure 6.

for the long flow is $40 \mathrm{Mb} / \mathrm{s}$; and the rates for the short flows are: $s_{1}=110 \mathrm{Mb} / \mathrm{s}, s_{2}=60 \mathrm{Mb} / \mathrm{s}$, and $s_{3}=s_{4}=40 \mathrm{Mb} / \mathrm{s}$. After the simulation starts, all flows converge to their maxmin rates within ten seconds. When flows $s_{5}$ to $s_{7}$ start at $t=20$, the bottleneck link for the long flow shifts to the $150 \mathrm{Mb} / \mathrm{s}$ link. The flow's rate quickly converges to its new max-min rate $30 \mathrm{Mb} / \mathrm{s}$. Correspondingly, the short flows $s_{1}$, $s_{5}, s_{6}$ and $s_{7}$ converge to their max-min rates $30 \mathrm{Mb} / \mathrm{s}$, and $s_{2}$ increases to its new max-min rate $70 \mathrm{Mb} / \mathrm{s}$, and $s_{3}$ and $s_{4}$

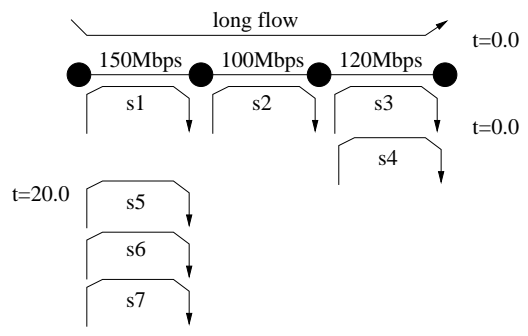

Fig. 9. The long flow and four short flows $s_{1}$ to $s_{4}$ start at time $t=0$. Three short flows $s_{5}, s_{6}$, and $s_{7}$ start at $t=20$. At time $t=40$, the short flows from $s_{4}$ to $s_{7}$ stop.

increase to $45 \mathrm{Mb} / \mathrm{s}$. At $t=40$, the short flows from $s_{4}$ to $s_{7}$ stop. The new bottleneck for the long flow is the $100 \mathrm{Mb} / \mathrm{s}$ link. It quickly converges to its max-min rate $50 \mathrm{Mb} / \mathrm{s}$. Similarly, $s_{1}$ converges to its new max-min rate $100 \mathrm{Mb} / \mathrm{s}, s_{2}$ converges to $50 \mathrm{Mb} / \mathrm{s}$, and $s_{3}$ converges to $70 \mathrm{Mb} / \mathrm{s}$.

Figure 12(a), 12(b) and 12(c) show how XCP's flow rate changes as bottleneck shifts. Figure 13(a), 13(b) and 13(c) show TCP's flow rates on each link. TCP cannot achieve maxmin fairness and its AIMD control algorithm leads to flow rates fluctuation. Additionally, fairness is an issue for TCP as flow's throughput is inversely proportional to its RTT, in the above topology, flows have different RTTs and considerable unfairness happens. For example, the long flow has the largest RTT and its flow is almost starved.

The convergence property of iXCP to equilibrium is similar to that of XCP. Both converge to equilibrium within seconds, whereas TCP persistently oscillates and never converges.

Another experiment we conducted is to test the convergence speed when $\mathrm{XXCP}$ and XCP converge to the same flow rates. We use the topology in Figure 1 and let four long flows passing both the $155 \mathrm{Mb} / \mathrm{s}$ link and the $100 \mathrm{Mb} / \mathrm{s}$ link without any short flows. The four long flows are all bottlenecked at the $100 \mathrm{Mb} / \mathrm{s}$ link with max-min fair flow rate $25 \mathrm{Mb} / \mathrm{s}$ each. Figure 14 (a) and Figure 14(b) show the flow rates and convergence speed for $\mathrm{iXCP}$ and XCP respectively. There is no noticable convergence speed difference between those two schemes.

\section{Varying RTTs}

We study how RTT values may impact the performance of iXCP using the topology shown in Figure 1. There are one short flow bottlenecked at the $155 \mathrm{Mb} / \mathrm{s}$ link and sixteen long flows bottlenecked at the $100 \mathrm{Mb} / \mathrm{s}$ link.

In the first set of experiments, all flows have the same RTTs, and we vary the RTTs from $40 \mathrm{~ms}$ to $1.4 \mathrm{~s}$. Figure $15(\mathrm{a}), 15(\mathrm{~b})$ and 15(c) show that iXCP preserves the good properties of $\mathrm{XCP}$ and is robust to large RTT values. Both $\mathrm{XXCP}$ and XCP maintain high link utilization and near-zero packet drops. This result is expected, because unlike TCP, both iXCP and XCP take into consideration the RTT value of a flow when adjusting a flow's congestion window. The average queue size of $\mathrm{XXCP}$ is larger than XCP because its link utilization is higher. Each flow also achieves its max-min rate for all RTT values we simulated. Next we measure the convergence time at which all the sixteen long flows reach a rate within $3 \%$ of its max-min fair rate. Figure 16 shows that the convergence time for both XCP and $\mathrm{XXCP}$ are almost linearly proportional to RTTs. And 


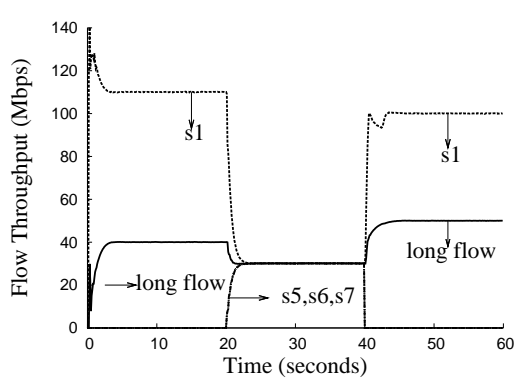

(a) Flow rates at the $150 \mathrm{Mb} / \mathrm{s}$ link

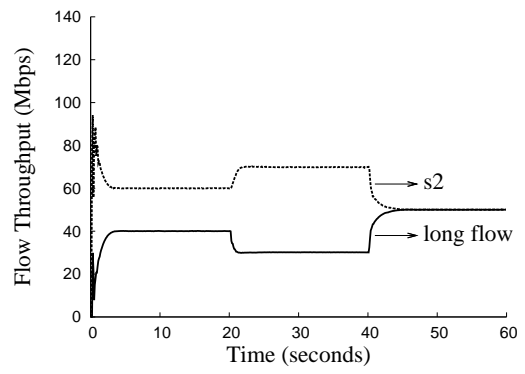

(b) Flow rates at the $100 \mathrm{Mb} / \mathrm{s}$ link

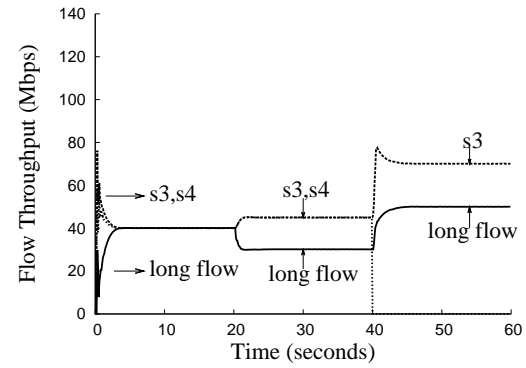

(c) Flow rates at the $120 \mathrm{Mb} / \mathrm{s}$ link

Fig. 11. These figures show how iXCP adapts to the flow dynamics of the network. The simulation topology is shown in Figure 9. Each flow can quickly converge to its max-min rate when other flows join or leave the network.

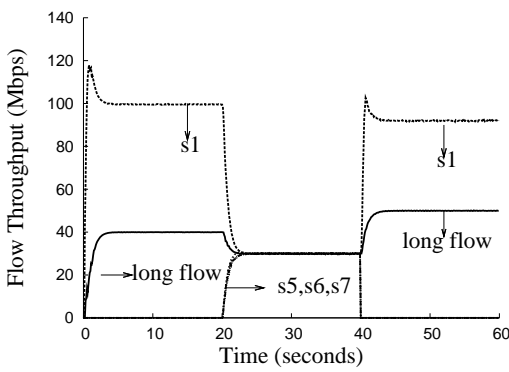

(a) Flow rates at the $150 \mathrm{Mb} / \mathrm{s}$ link

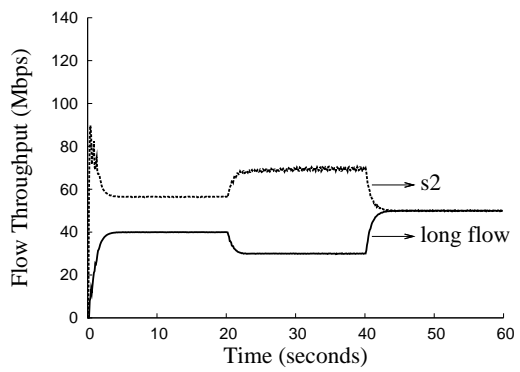

(b) Flow rates at the $100 \mathrm{Mb} / \mathrm{s}$ link

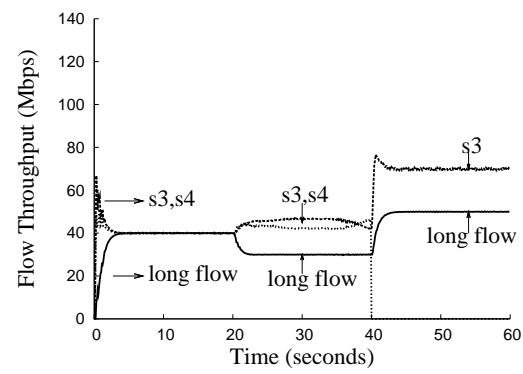

(c) Flow rates at the $120 \mathrm{Mb} / \mathrm{s}$ link

Fig. 12. These figures show how XCP adapts to the flow dynamics of the network. The simulation topology is shown in Figure 9. Each flow can quickly converge to its equilibrium rate when other flows join or leave the network. However, some flows' equilibrium rates in XCP are smaller than that in iXCP.

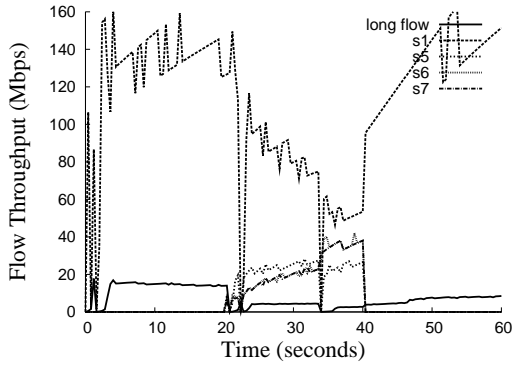

(a) Flow rates at the $150 \mathrm{Mb} / \mathrm{s}$ link

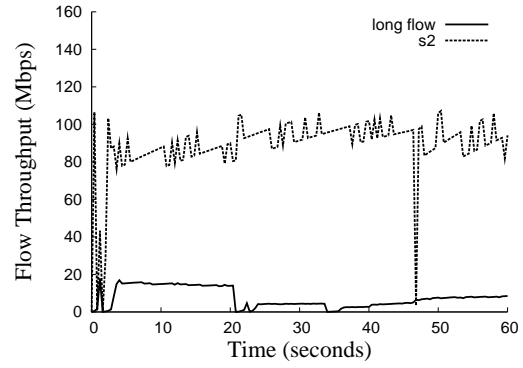

(b) Flow rates at the $100 \mathrm{Mb} / \mathrm{s}$ link

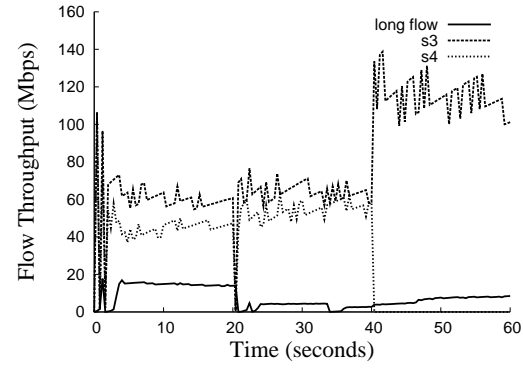

(c) Flow rates at the $120 \mathrm{Mb} / \mathrm{s}$ link

Fig. 13. These figures show how TCP performs in dynamic case. The simulation topology is shown in Figure 9. TCP flows oscillates and never converges. TCP cannot achieve fairness among its flows.

iXCP's convergence time is slightly higher than XCP because iXCP needs some time for its bottleneck id to be correctly identified.

In the second set of experiments, flows have heterogeneous RTT values. The purpose is to study whether iXCP ensures fairness among flows with heterogeneous RTTs. In our experiments, the short flow has a 40ms RTT value. The other sixteen long flows have RTTs ranging from $60 \mathrm{~ms}$ to $1.26 \mathrm{~s}$ with an $80 \mathrm{~ms}$ increment. Figure 17 shows the ratio between a flow's rate and its theoretical max-min rate. The flow with ID 0 is the short flow and the flows with IDs from 1 to 16 are the sixteen long flows. With iXCP, all flows achieve their max-min rates regardless of their RTT values. With XCP, the sixteen long flows achieve max-min fair rates; but the short flow does not obtain its max-min rate due to the under-utilization problem stated previously (Section II-B).

\section{E. Web-like Traffic}

We study the impact of flow dynamics on the performance of iXCP using web-like traffic. We use the two-bottleneck topology as shown in Figure 1. The long-lived background traffic includes the short flow that crosses the $155 \mathrm{Mb} / \mathrm{s}$ link and sixteen long flows that cross both the $155 \mathrm{Mb} / \mathrm{s}$ link and the $100 \mathrm{Mb} / \mathrm{s}$ link. The short-lived web-like traffic crosses both the $155 \mathrm{Mb} / \mathrm{s}$ link and the $100 \mathrm{Mb} / \mathrm{s}$ link. These flows arrive according to a Poisson process with arrival rates in the range of [10/sec, 1000/sec]. The transfer size of these flows is derived from a Pareto distribution with an average of 30 packets (ns implementation with shape $\_=1.35$ ), which is consistent with real web traffic study [15].

Figure 18(a), 18(b) and 18(c) show the link utilization, average queue size and packet drops on the $155 \mathrm{Mb} / \mathrm{s}$ link and the $100 \mathrm{Mb} / \mathrm{s}$ link for iXCP and XCP.

As can be seen, both XCP and iXCP's performance are less than ideal in highly dynamic situations: link utilization 


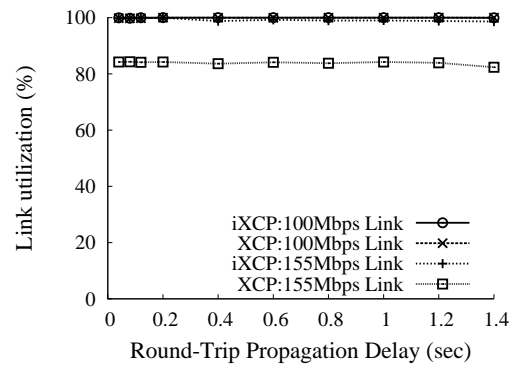

(a) Link utilization

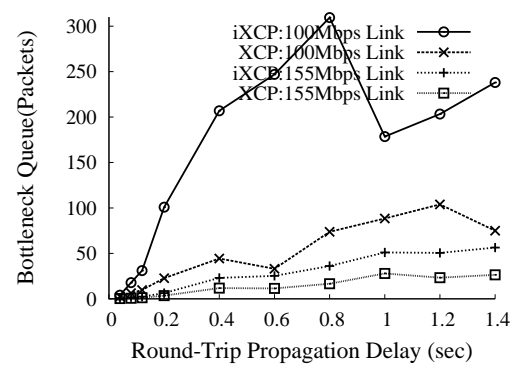

(b) Average queue size

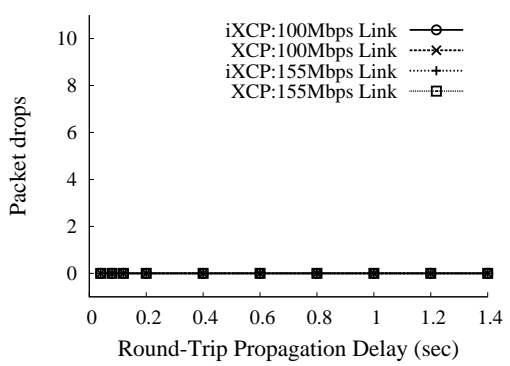

(c) Packet drops

Fig. 15. iXCP preserves the good property of XCP and is robust to large RTT values. It maintains high link utilization, low average queue size, and zero packet drops.

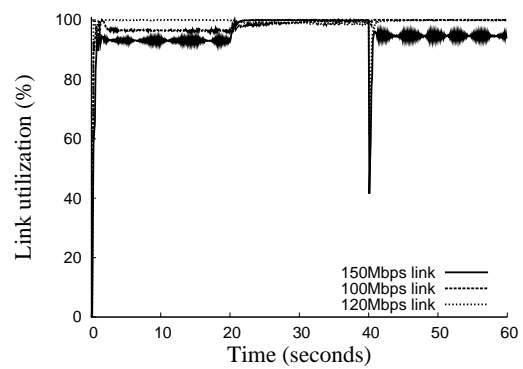

(a) Link utilization of XCP

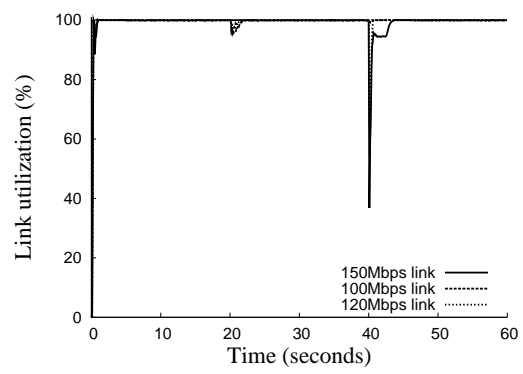

(b) Link utilization of iXCP

Fig. 10. XCP under-utilizes some links, while iXCP achieves nearly $100 \%$ link utilization for all the three links, as shown in Figure 9.

decreases, queue size and packet drops increase. iXCP outperforms XCP on both links. The performance degrades because web-like traffic exits the network before the flow allocation in the network has reached equilibrium. When a new flow joins the network, iXCP and XCP deallocate bandwidth from the existing flows to the new flow. Before the existing flows converge to their new rate allocations, bandwidth may be temporarily over-allocated, leading to congestion. When a flow departures from the network, the bandwidth allocated to the flow may be temporarily wasted before the network can assign it to other flows, causing link under-utilization. As the control algorithm of both $\mathrm{XXCP}$ and XCP assigns positive window increment inversely proportional to a flow's rate, a new flow with a small starting rate has a larger window increment than a flow that departures the network. Therefore, the over-allocation and the under-utilization effects can not exactly cancel out, even though the departure rates and the arrival rates of the web-like flows are the same. As shown in Figure 18(a), 18(b) and $18(\mathrm{c})$ ), for the $100 \mathrm{Mb} / \mathrm{s}$ link, when the arrival rates of the web-like flows are high $(>400 / \mathrm{sec})$, the over-allocation

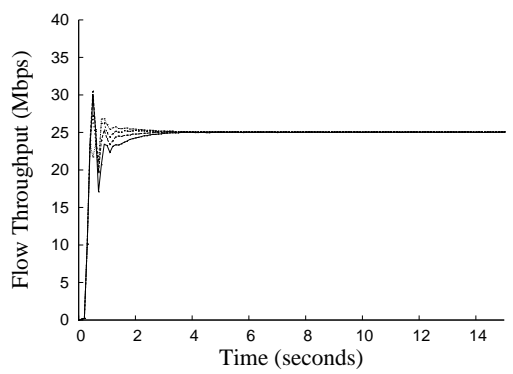

(a) iXCP flows.

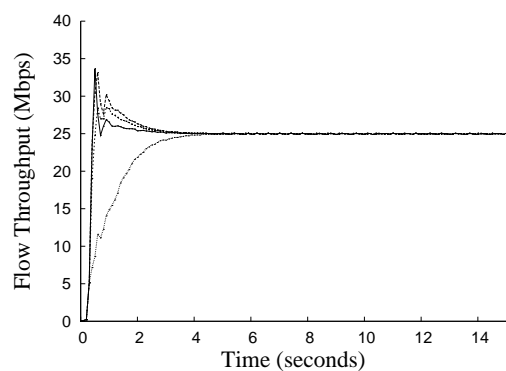

(b) XCP flows.

Fig. 14. iXCP and XCP exhibit similar convergence speed when their flows converge to the same max-min fair rates.

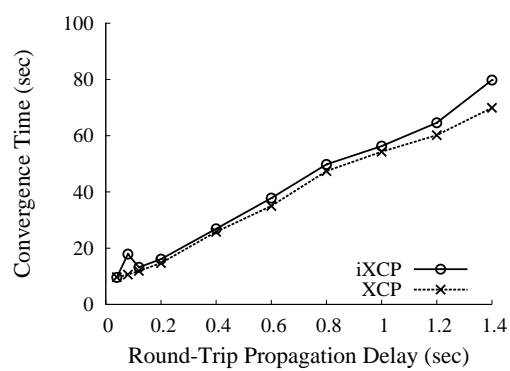

Fig. 16. Both XCP and iXCP convergence time is almost linearly proportional to RTTs.

effect dominates. The link is fully utilized, but the queue size increases and packet drops occur. When the arrival rate is low $(\leq 400 / \mathrm{sec})$, the departure effect dominates, and the link is under-utilized. For the $155 \mathrm{Mb} / \mathrm{s}$ link, the link remains underutilized when the arrival rates are high, because the overallocation to the new web-like flows prevents the short flow from reaching its equilibrium rate.

The link utilization for iXCP will increase when the number of short flows increases, as this will reduce the over-allocation 


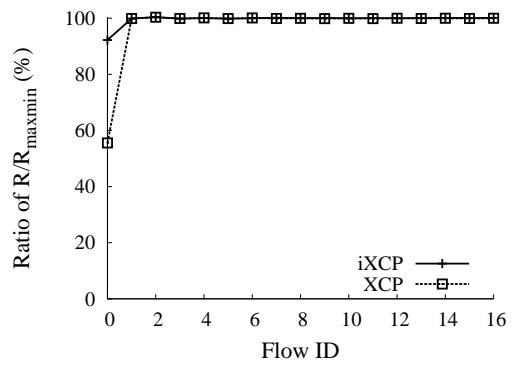

Fig. 17. iXCP is max-min fair to all flows even in the presence of highly heterogeneous RTTs.

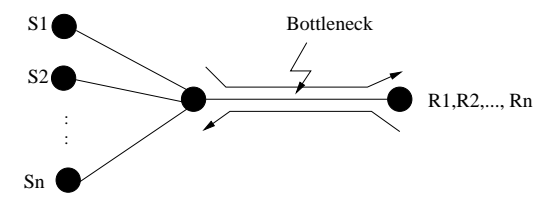

Fig. 21. A single bottleneck topology

to the web-like flows. Figure 19(a), 19(b) and 19(c) show the simulation results when there are ten short flows passing the first $155 \mathrm{Mb} / \mathrm{s}$ link. More performance improvements of iXCP over XCP are demonstrated when the arrival rates of web traffic are high.

We have conducted another experiment where each link has web traffic across it and only across it, i.e., at the $155 \mathrm{Mb} / \mathrm{s}$ link, the short flow is mixed with a set of web traffic; at the $100 \mathrm{Mb} / \mathrm{s}$ link, the sixteen long flows are mixed with another set of web traffic. In this scenario, both the short flow and long flows are greatly impacted by web traffic. Figure 20(a), 20(b) and 20(c) show the simulation results for this case. When arrival rate of web traffic is high, both links are dominated by the short-lived web traffic and over-allocation effect leads to high utilization, larger queue size and more packet drops.

It is our future work to further investigate how flow dynamics interact with the control algorithm of XCP and iXCP. Is there a lower bound on the link utilization in dynamic situations? Can the performance of iXCP be further improved?

\section{F. Single-link topology}

We use the single-link topology and the parking-lot topology as in [8] to perform sanity check for iXCP scheme. The simultion scenarios cover those with different link capacity, different round-trip delay, dynamic case with new traffic joining and the case with web traffic introduced. The performance metrics we are interested are link utilization, average queue size, packet drop and flow rate.

In this topology, 50 flows traverse the single link in the forward direction and 50 flows traverse in the reverse direction to stress iXCP and XCP.

1) Impact of bandwidth: single link bandwidth is increased from $50 \mathrm{Mbps}$ to $2 \mathrm{Gbps}$. Link propagation delay is $80 \mathrm{~ms}$. Figure 22(a), Figure 22(b) and Figure 22(c) show that both iXCP and XCP are efficient with link capacity increases and can achieve high utilization, low queue size and zero packet drop.

2) Impact of RTT: We fix the link bandwidth to be $150 \mathrm{Mbps}$ and vary the link propagation delay to make flows

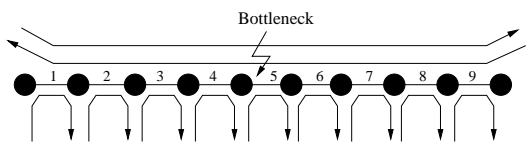

Fig. 26. A parking lot topology

have different RTTs. Figure 23(a), Figure 23(b) and Figure 23(c) show that both iXCP and XCP can achieve high utilization, low queue size and zero packet drop, robust to round-trip time increases.

3) Convergence Dynamics: In this experiment, link capacity is set to be $45 \mathrm{Mbps}$ and RTT is $40 \mathrm{~ms}$. Five long-lived flows share the single link and flows start at different times, 0,2 , 4,6 8 seconds.

Figure 24(a) shows that whenever there is a new flow joining, both XCP and iXCP can reallocate a new max-min fair rate to each flow. Figure 24(b) and Figure 24(c) show that during convergence, utilization is not disturbed; queue can accomodate traffic burst and drain afterwards.

4) Impact of web traffic: We introduce web-like traffic into our simulations to study the impact of flow dynamics on the performance of $\mathrm{XXCP}$ and XCP in single-link topology. The web traffic arrives according to a Poisson process with arrival rates in the range of $[10 / \mathrm{sec}, 1000 / \mathrm{sec}]$. The transfer size of these flows is derived from Pareto distribution with an average of 30 packets, which is consistent with real web traffic study [15].

Figure 25(a), 25(b) and 25(c) show the link utilization, average queue size and packet drops on the single link for iXCP and XCP.

The utilization for the single link is similar in both $\mathrm{XXCP}$ and XCP. This is because our modification to XCP only affects the rate allocation when link under-utilization problem exists. In a single-link topology, we do not have this problem.

\section{G. Parking-lot Topology}

In the parking lot topology, there are total nine links. Each link has capacity $100 \mathrm{Mb} / \mathrm{s}$, except link 5 has $50 \mathrm{Mb} / \mathrm{s}$. There are 50 long flows traversing all the links in forward direction and 50 long flows in the reverse direction. At each link, there are 50 flows across only that link. Thus, long flows are all bottlenecked at link 5. As we explained before, in this multi-bottleneck topology, link under-utilization problem exists for each link. For the first four links, long flows are bottlenecked at downstream links, however, upstream links still attempt to increase long flows' rates but they cannot increase any further. Thus, iXCP can increase long flows' rates and iXCP outperforms XCP in terms of link utilization (reverse traffic stresses the schemes and makes it not achieve $100 \%$ utilization). For the last four hops, long flows are bottlenecked at upstream link, in XCP's implementation, residual terms help to save some bandwidth for the bottleneck flows, but it does not solve the link under-utilization problem and cannot achieve $100 \%$ utilization.

\section{H. Comparison of $i X C P, P-X C P$ and JetMax}

In this section, we compare iXCP with P-XCP [2] and JetMax [3]. Zhou et al. also forsaw XCP's problem and proposed 


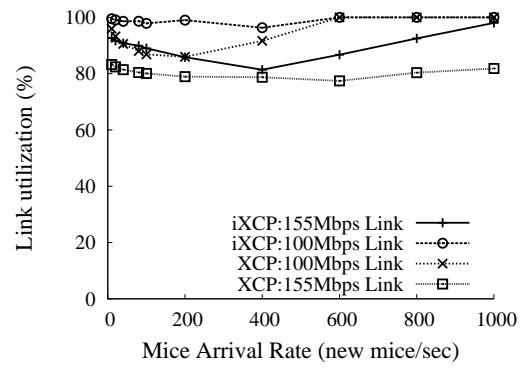

(a) Link utilization

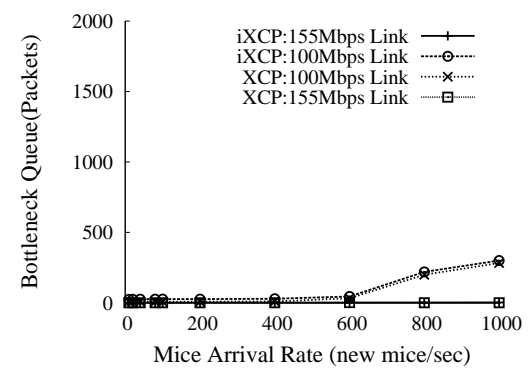

(b) Average queue size

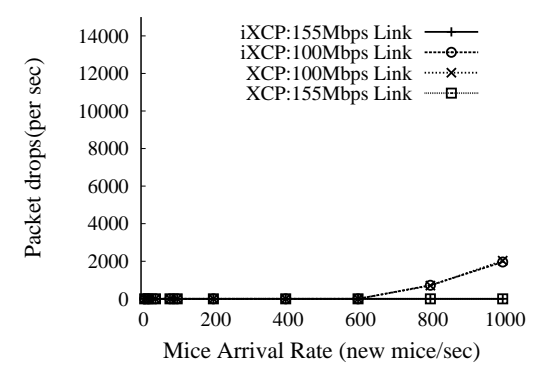

(c) Packet drops

Fig. 18. iXCP outperforms XCP in a highly dynamic environment with the arrivals and departures of web-like traffic.

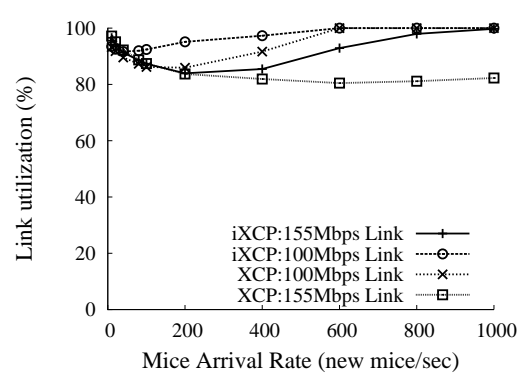

(a) Link utilization

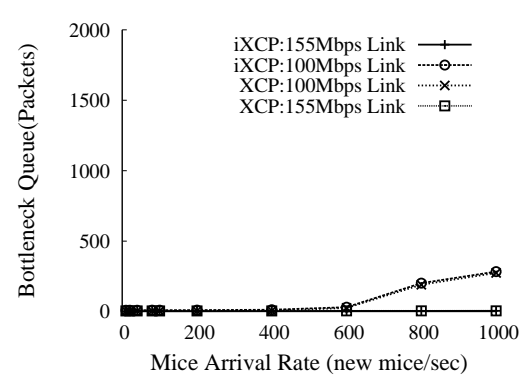

(b) Average queue size

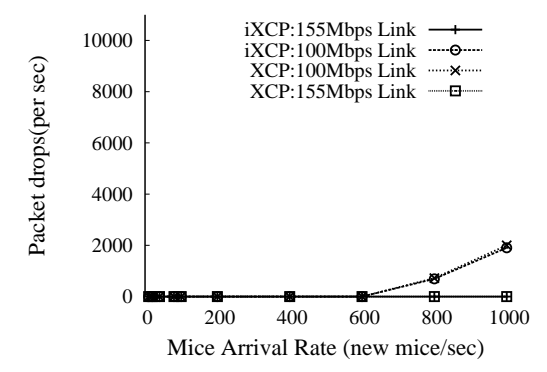

(c) Packet drops

Fig. 19. iXCP achieves more improvment over XCP if we increase the number of short flows to 10 at the $155 \mathrm{Mb} / \mathrm{s}$ link, in a highly dynamic environment with the arrivals and departures of web traffic.

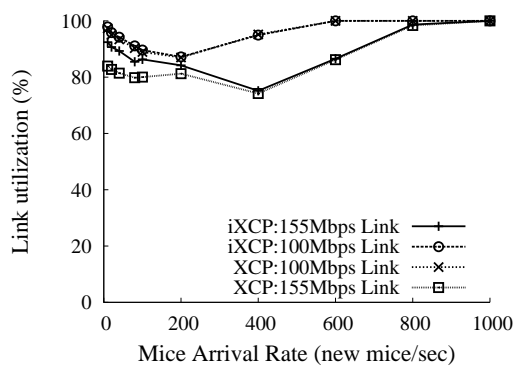

(a) Link utilization

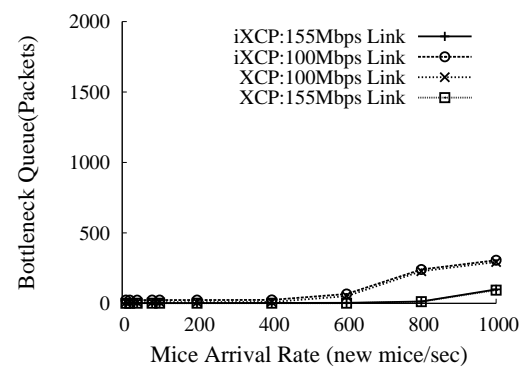

(b) Average queue size

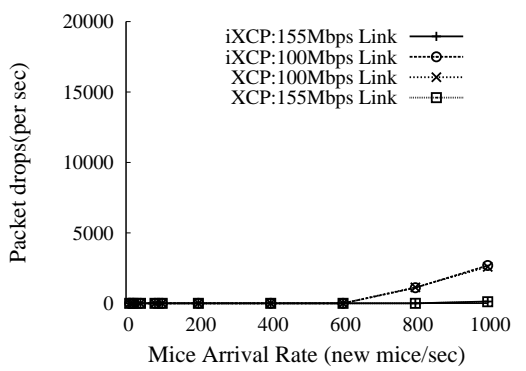

(c) Packet drops

Fig. 20. In this simulation, each link has web traffic traverse it. Thus, the single short flow is mixed with web traffic at the $155 \mathrm{Mb} / \mathrm{s}$ link and the sixteen long flows are mixed with another set of web traffic at the $100 \mathrm{Mb} / \mathrm{s}$ link. As web traffic dominates both links when arrival rate of web traffic is high, the $155 \mathrm{Mb} / \mathrm{s}$ link utilization approaches to that of the $100 \mathrm{Mb} / \mathrm{s}$ link.

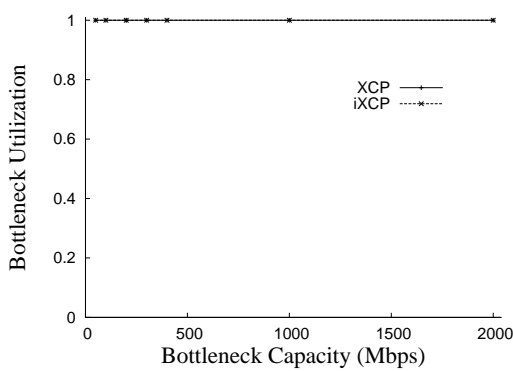

(a) Link utilization

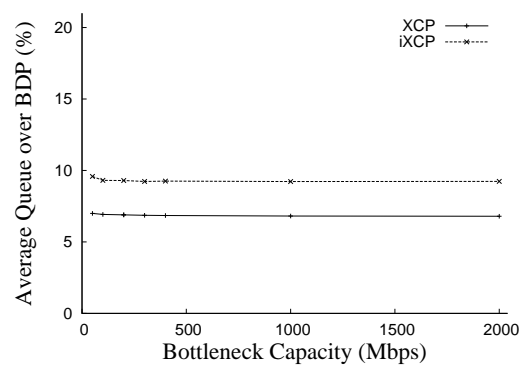

(b) Average queue size

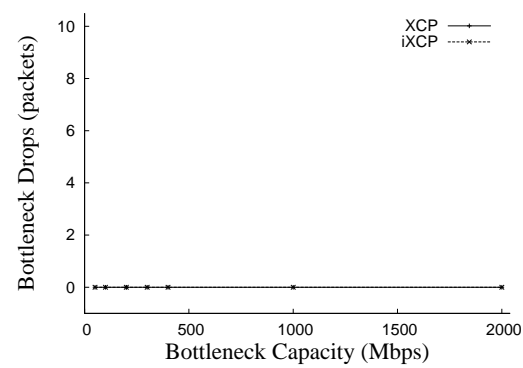

(c) Packet drops

Fig. 22. Both iXCP and XCP have high link utilization, low average queue size and zero drop at single-link with different link bandwidth.

$\mathrm{P}-\mathrm{XCP}$ [2] to improve XCP's under-utilization problem. With $\mathrm{P}-\mathrm{XCP}$, a router estimates the number of bottlenecked flows by comparing the feedback in a packet header with the one it computes. If the computed feedback is smaller, then the router considers the flow as bottlenecked at itself. The router allocates more bandwidth to those flows that are bottlenecked at itself by scaling the spare bandwidth with a factor $N / N_{b}$, in which $N$ is the total number of flows, and $N_{b}$ is the number of flows that are bottlenecked at the router. This scheme only increases link utilization when a bottleneck link is upstream to an under-utilized link. For instance, it does not increase link utilization in the example shown in Figure 1. Moreover, as P$\mathrm{XCP}$ over-allocates spare bandwidth, it causes rate fluctuations and increases the average queue size.

JetMax [3] is a rate-based congestion control algorithm that aims to provide max-min fairness. It also includes a bottleneck 


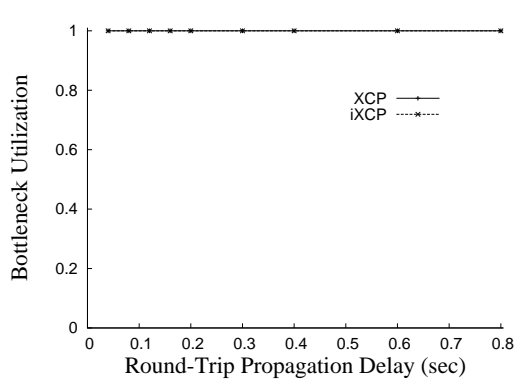

(a) Link utilization

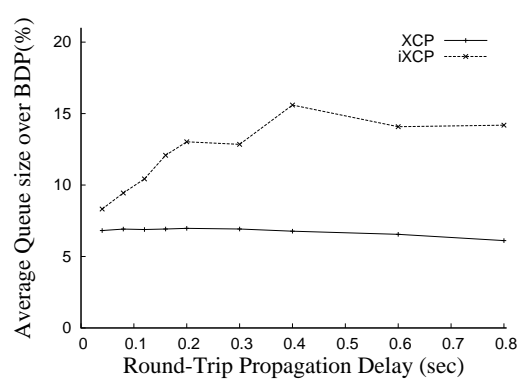

(b) Average queue size

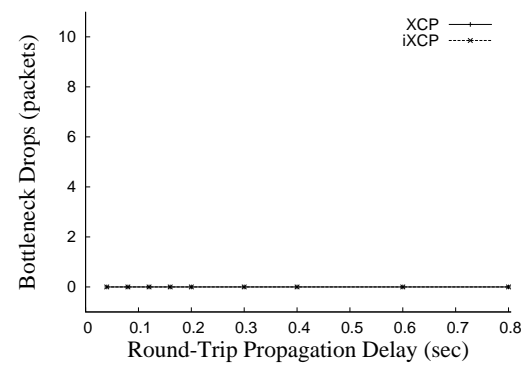

(c) Packet drops

Fig. 23. Both iXCP and XCP have high link utilization, low average queue size and zero drop at single-link with different RTTs.

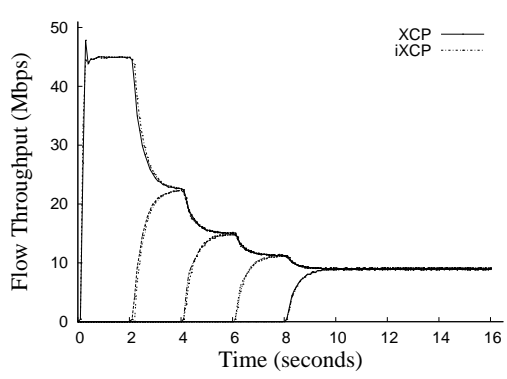

(a) Flow rate

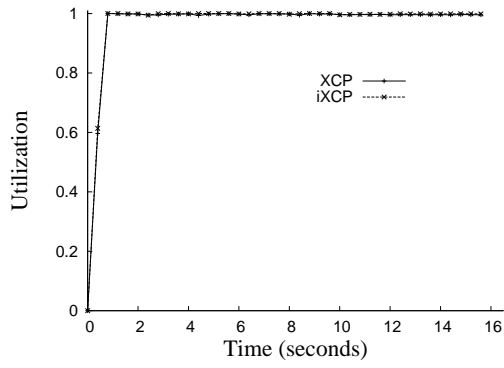

(b) Link utilization

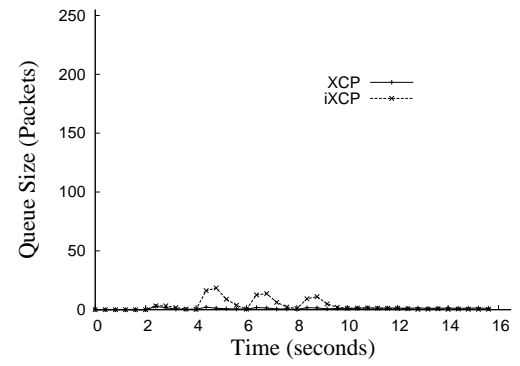

(c) Average queue size

Fig. 24. Both iXCP and XCP smoothly converge to high fairness, good utilizationa and small queue size. Five flows start at times $0,2,4,6,8$ respectively.

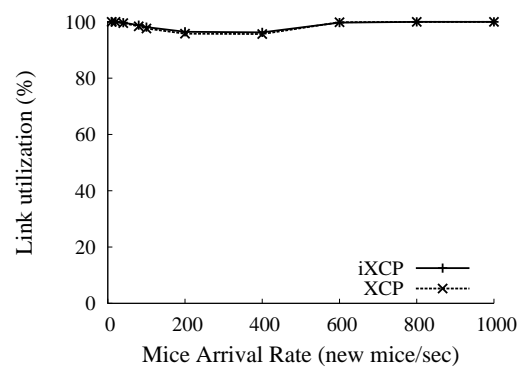

(a) Link utilization

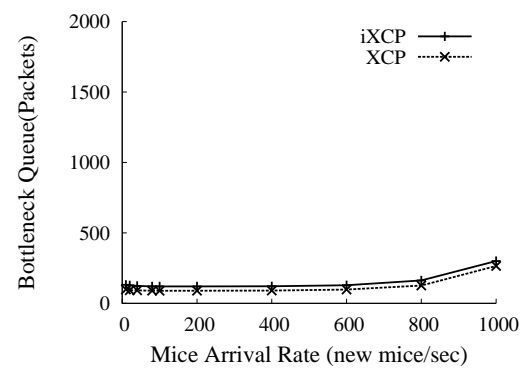

(b) Average queue size

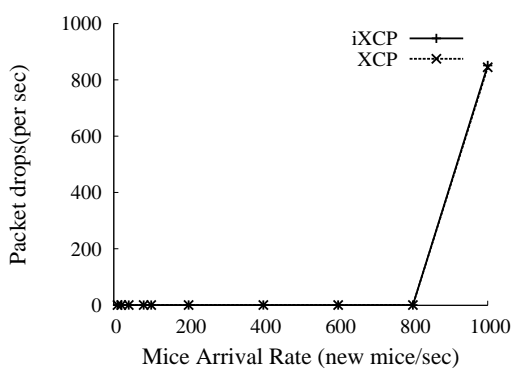

(c) Packet drops

Fig. 25. Both iXCP and XCP are robust and efficient in highly dynamic environments with arrivals and departures of web traffic.

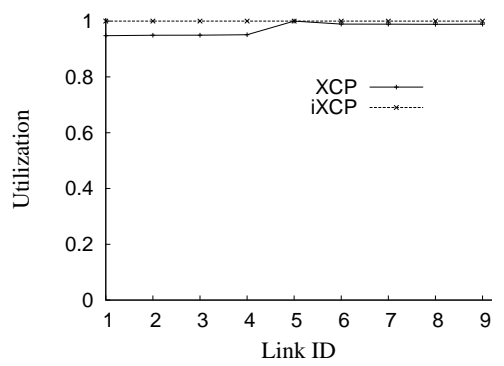

(a) Link utilization

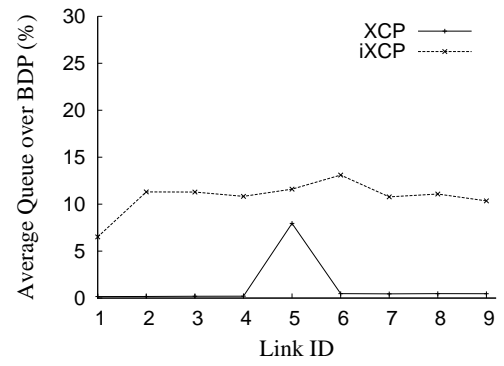

(b) Average queue size

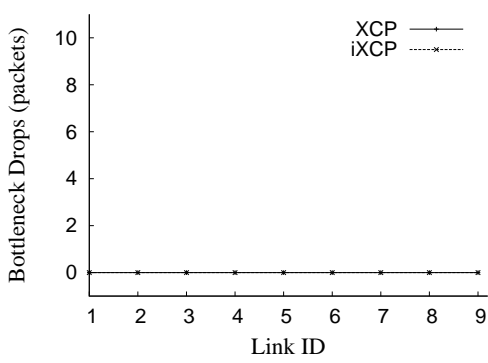

(c) Packet drops

Fig. 27. Simultion of iXCP and XCP in multi-bottleneck parking-lot topology.

identifier field in a packet to facilitate max-min rate allocation. Different from XCP and iXCP, JetMax directly returns the max-min fair rate to a flow, instead of the rate adjustment.

In the first set of experiments, we compare whether they can achieve max-min fair allocation on the topology shown in Fig. 1 where one short flow traverses the $155 \mathrm{Mb} / \mathrm{s}$ link and four long flows traverse both the $155 \mathrm{Mb} / \mathrm{s}$ link and the $100 \mathrm{Mb} / \mathrm{s}$ link. Fig. 28(a) shows the results. Both iXCP and JetMax can achieve max-min fair allocation to the short flow, but the convergence time for JetMax is slightly longer than
iXCP. P-XCP cannot allocate to the short flow its fair share of bandwidth, because the upstream router does not know how many flows are really bottlenecked at it. When we switch the two links' position in Fig. 28(a) and run the simulations again, $\mathrm{P}-\mathrm{XCP}$ is able to increase short flow's sending rates, as shown in Fig. 28(b). However, from Fig. 28(b) and Fig. 28(c), it can be seen that P-XCP causes rate fluctuations and increases the average queue size because it over-allocates a link's spare bandwidth.

In the second set of the experiments, we compare iXCP 


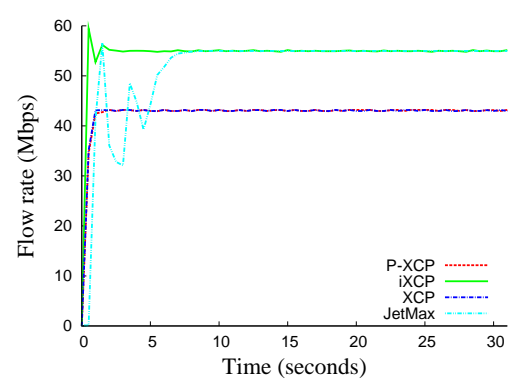

(a) Short flow rate

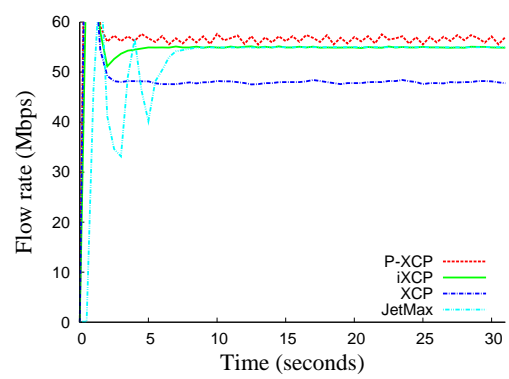

(b) Short flow rate in reverse topology

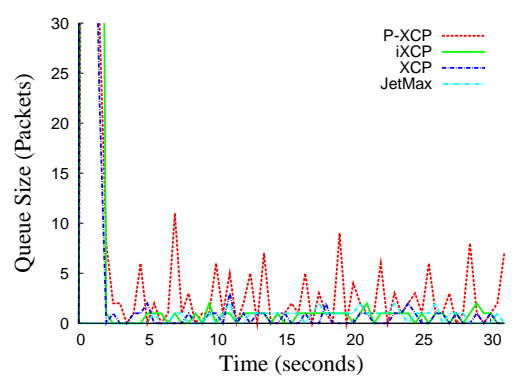

(c) Queue size in reverse topology

Fig. 28. Comparing to iXCP, XCP and JetMax, P-XCP can overshoot link bandwidth and lead to rate fluctuation and queue size increase.

with JetMax. We first compare XCP, iXCP and JetMax's performance under the topology $\mathcal{T}_{2}$ shown in Fig. 29(a). This is the topology used in [3]. In this topology, nodes $R_{1}$ and $R_{4}$ route their traffic (including any ACKs) in the clockwise direction. Flow $x_{1}$ to $x_{4}$ starts one after the other with 30 seconds apart. We refer to flow $x_{1}$ and $x_{3}$ as long flow and flow $x_{2}$ and $x_{4}$ as short flow. Figure 29(b), 29(c), and 30(a) show the rates of four flows with respective to time for $\mathrm{XCP}$, JetMax and iXCP respectively. As we can see, flow rates in $\mathrm{XCP}$ and $\mathrm{XXCP}$ oscillate in this case, while JetMax achieves a stable max-min rate allocation. Because XCP and iXCP oscillate for the same reason, we therefore only elaborate on iXCP. Figure 31(a) shows that all bottlenecked links are fully utilized despite rate oscillation. Moreover, in Figure 32(a) and Figure 34(a), the window sizes of all flows are relatively stable, showing the saw-toothed behavior of a window-based flow control protocol. Figure 33(a) and Figure 35(a) show the RTTs of each flow in iXCP. As can be seen, a flow's rate fluctuation is mostly caused by its RTT fluctuation. Despite this fluctuation, the long term average rate of a flow is still max-min fair.

The RTT oscillation problem in XCP and $\mathrm{XCP}$ is primarily caused by ACK compression and heterogeneous RTTs. As a short flow only has a 20ms RTT, the random queuing delays caused by ACKs is orders of magnitudes larger than the short flow's RTT. As a flow's rate is computed as the window size divided by RTT, a large variation in RTTs leads to a large variation in the flow's sending rate. In addition, in this scenario, the long and short flows' RTTs differ significantly and the router's control interval, which is estimated as the average RTT among all flows, is less than half of the long flow's RTT. Within one RTT, a long flow may receive positive feedback in one control interval, and negative feedback in another control interval. Because of this inconsistent feedback, a flow can not smooth out its sending rates using the natural ACK clocking of a window-based protocol. Instead, a long flow sends faster when it receives positive feedback in one control interval, and slower when it receives negative feedback. This fluctuation causes the queuing delay to fluctuate, which causes the short flow's sending rate to oscillate. The fluctuation caused by large RTT variance is also observed and explained by $[16]$.

To confirm this analysis, we first implement a priority queue for ACK packets to smooth the random queuing delay caused by ACKs. We then increase the router's control interval to the maximum RTT among all flows to smooth the long flows' sending rates. Figure 30(b) and Figure 30(c) show the results. As can be seen from Figure 33(b) and Figure 32(b), after we use the priority queue, both RTT and window fluctuation of short flows are significantly reduced. From Figure 33(c) and Figure 32(c), after we extend the router's control interval, this fluctuation becomes negligible. As a results, all flows' rates converge to their max-min fair shares. The results for XCP is similar so that we do not present them here.

We next compare JetMax with iXCP in the Web-like Traffic scenarios as shown in the first configuration of Section IV-E. We ommit the results in other configurations since the results are similar. Fig. 36(a) shows JetMax achieve as low as $40 \%$ of link utilization in some situations. Fig. 36(b) and Fig. 36(c) compare the average queue size and packet loss rate. We can see that when the input web traffic rate is high, JetMax experiences a higher average queue size and higher packet loss rate. There are three reasons for these effects. First, Jetmax delayed response to rate adjustment by $R T T+(1+k) \Delta$, where $\Delta$ is the control interval and $0 \leq k \leq 1$, after bottleneck switch. And short-lived flow may terminate during the interval, not able to adjust their rate. Second, even though a Jetmax flow, after bottleneck switch, can overcome the false calcuation of number of bottlenecked flows caused by its own switch, it cannot overcome the false calculation due to other flows' arrival and leave. Third, short-lived Jetmax flows are not able to fully utilize the bandwidths allocated to them because endusers use exponential weighted moving average to adjust rate. The under-utilization problem of Jetmax in the face of mice traffic is also identified and explained by [17]. XCP or iXCP is more robust because its feedback is the relative windowsize adjustment, and not the absolute window size. If a link is under utilized, some flows will receive positive feedback, and increase their sending rates in the next round trip time. 


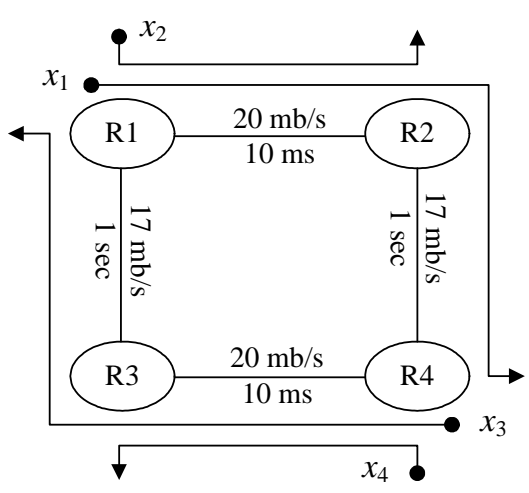

(a) JetMax $\mathcal{T}_{2}$ Topology

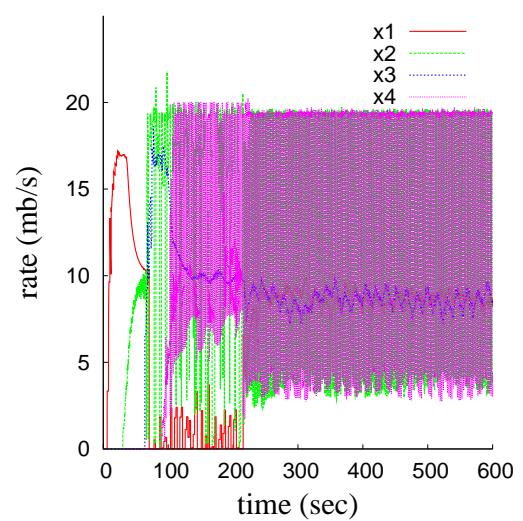

(b) XCP flow rate

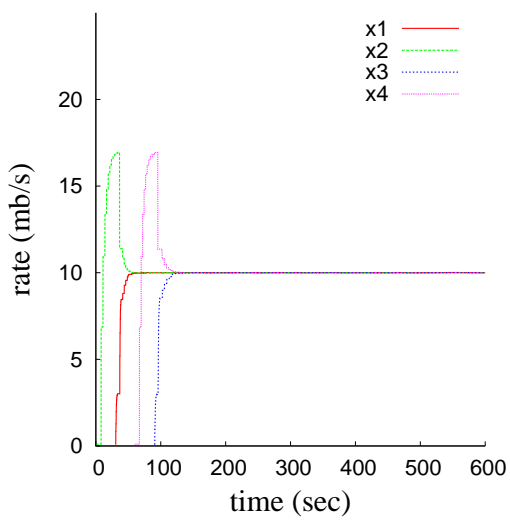

(c) JetMax flow rate

Fig. 29. XCP and JetMax flow rate in JetMax $\mathcal{T}_{2}$ topology.

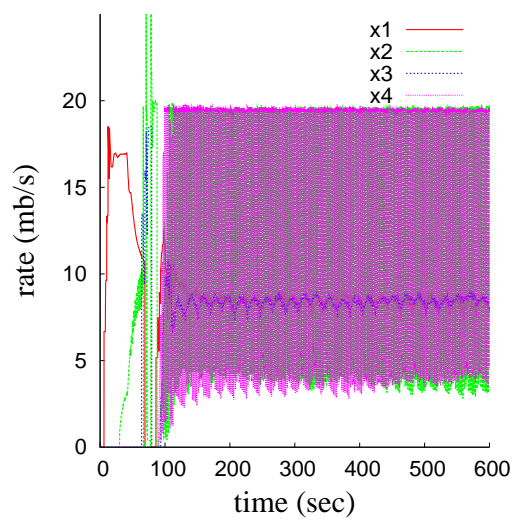

(a) iXCP flow rate without ACK priority queue

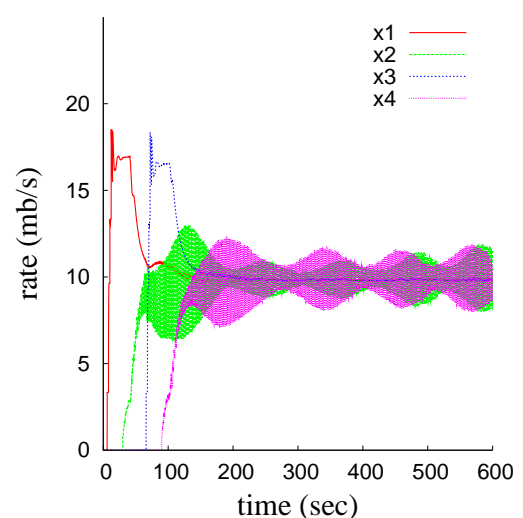

(b) iXCP flow rate with ACK priority queue

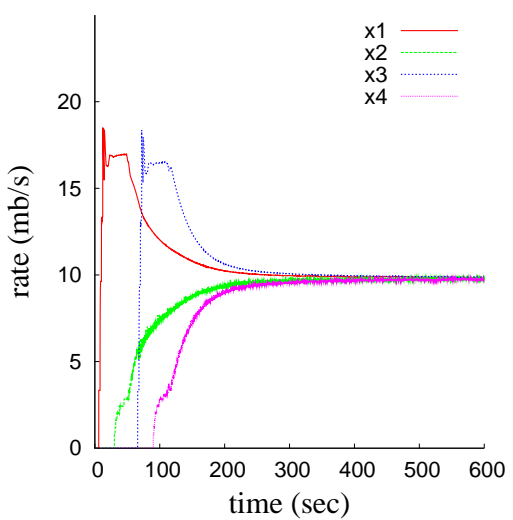

(c) iXCP flow rate with ACK priority queue and estimation-control interval equal to max RTT

Fig. 30. iXCP flow rate in JetMax $\mathcal{T}_{2}$ topology.

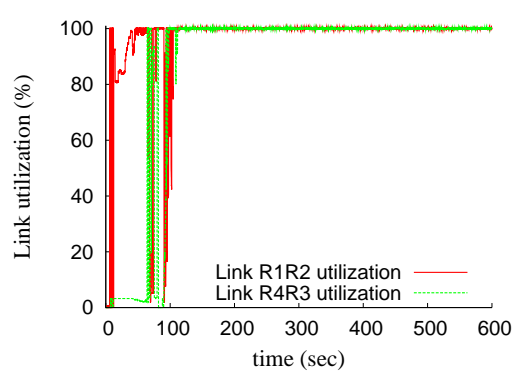

(a) iXCP link utilization without ACK priority (b) queue

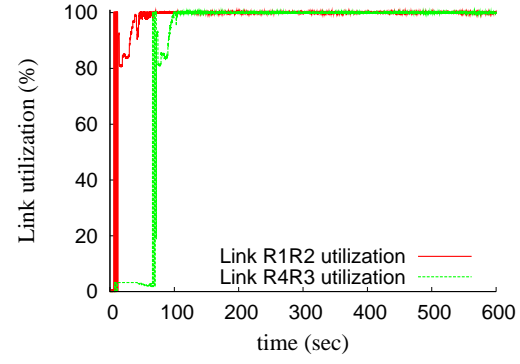

queue

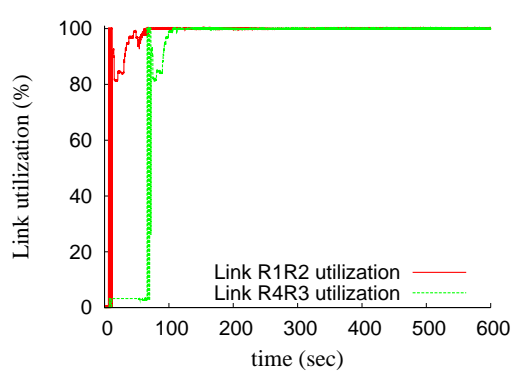

(c) iXCP link utilization with ACK priority queue and estimation-control interval equal to max RTT

\section{Fig. 31. iXCP link utilization in JetMax $\mathcal{T}_{2}$ topology.}

\section{Summary}

In all our simulations except the simulations for web traffic, iXCP achieved a near optimal bandwidth allocation: $100 \%$ link utilization with max-min fairness, as predicated by our theoretical analysis (Section A). iXCP also preserves all the good properties of the original XCP, including small average queue sizes, near-zero packet drops, and fast convergence. In the case where flows' RTTs differ significantly, XCP's flow rates may oscillate, but it can still achieve a high link utilization. In a highly dynamic environment where a large number of short-lived flows depart and arrive rapidly in the network, flows cannot reach their equilibrium rates and thus iXCP's improvement degrades, but it still outperforms XCP and JetMax in terms of link utilization.

\section{RELATED WORK}

Low et al. simulated and analyzed XCP's under-utilization problem [10]. This work is inspired by their discovery. Our analysis is built on Low's model. Zhou et al. also foresaw this problem and proposed $\mathrm{P}-\mathrm{XCP}$ [2]. However, P-XCP does not address the root cause of the under-utilization problem, and only alleviates the problem in some topologies. With P$\mathrm{XCP}$, a router estimates the number of bottlenecked flows by comparing the feedback in a packet header with the one it computes. If the computed feedback is smaller, then the router considers the flow as bottlenecked at itself. The router 


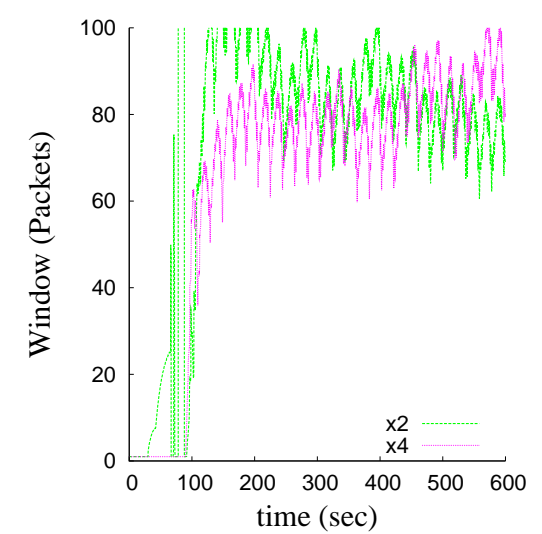

(a) iXCP short flow's window size without ACK priority queue

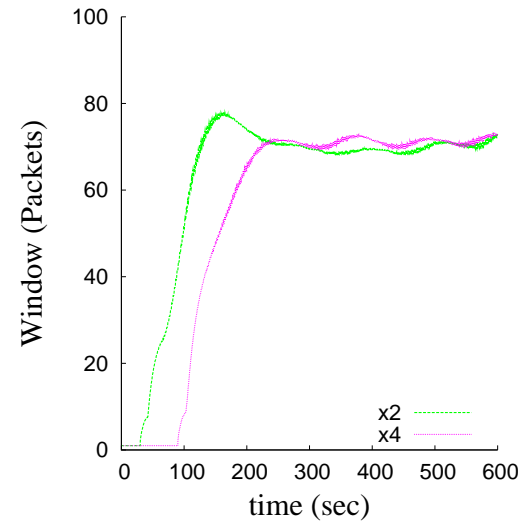

(b) iXCP short flow's window size with ACK priority queue

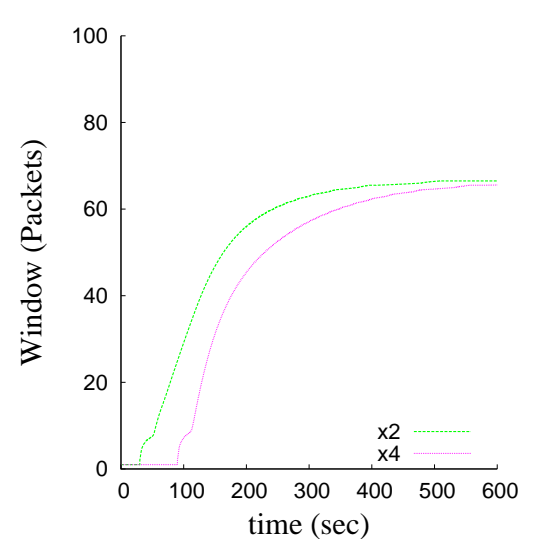

(c) iXCP short flow's window size with ACK priority queue and estimation-control interval equal to max RTT

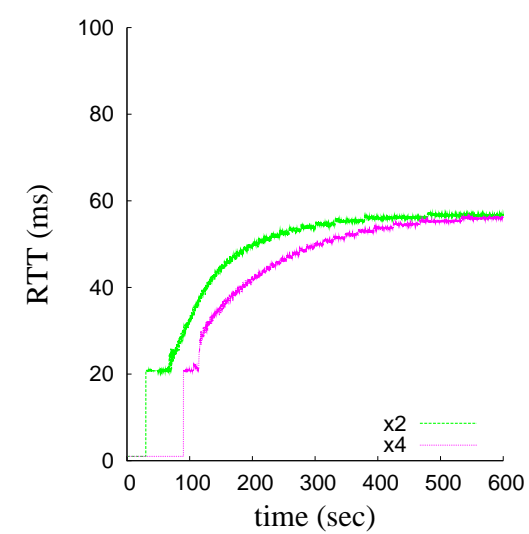

(c) iXCP short flow's RTT with ACK priority queue and estimation-control interval equal to max RTT

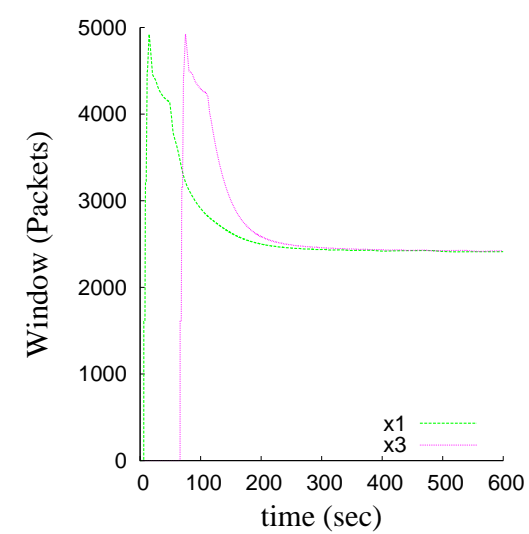

(c) iXCP long flow's window size with ACK priority queue and estimation-control interval equal to max RTT

Fig. 34. iXCP long flow window size in JetMax $\mathcal{T}_{2}$ topology.

allocates more bandwidth to those flows that are bottlenecked at itself by scaling the spare bandwidth with a factor $N / N_{b}$, in which $N$ is the total number of flows, and $N_{b}$ is the flows that are bottlenecked at the router. This scheme only increases link utilization when a bottleneck link is upstream to an under-utilized link. For instance, it does not increase link utilization in the example shown in Figure 1. Moreover, as P-
XCP over-allocates spare bandwidth, it causes rate fluctuations and increases the persistent queue size.

To the best of our knowledge, our work is the first that systematically addresses the under-utilization problem of XCP and to prove that the improved XCP is max-min fair in all types of topologies in steady state.

JetMax [3] is a rate-based congestion control algorithm that 


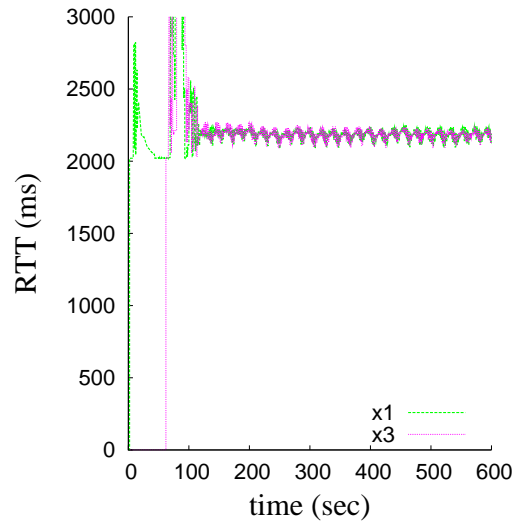

(a) iXCP long flow's RTT without ACK priority queue

Fig. 35. iXCP long flow RTT in JetMax $\mathcal{T}_{2}$ topology.

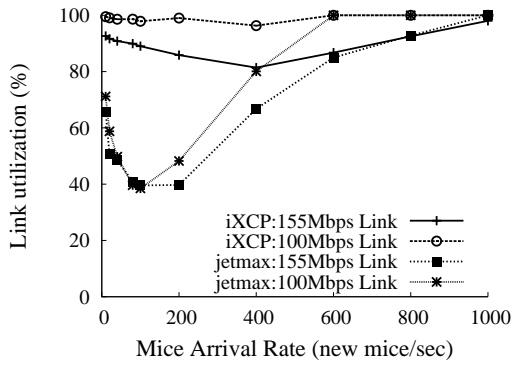

(a) Link utilization

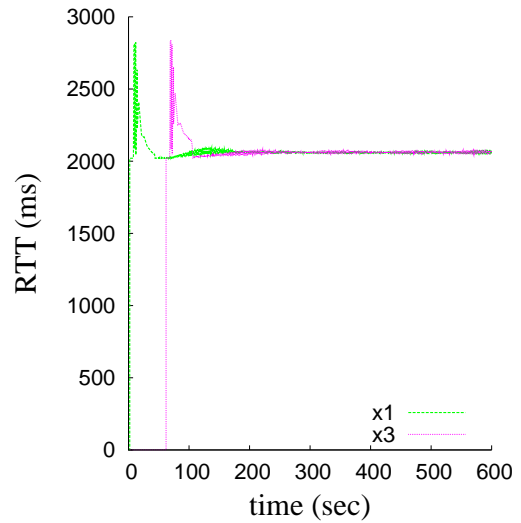

(b) iX
queue

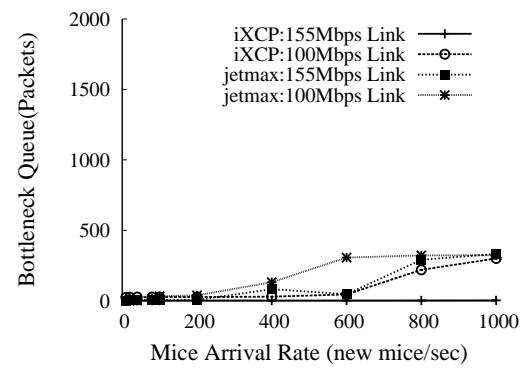

(b) Average queue size

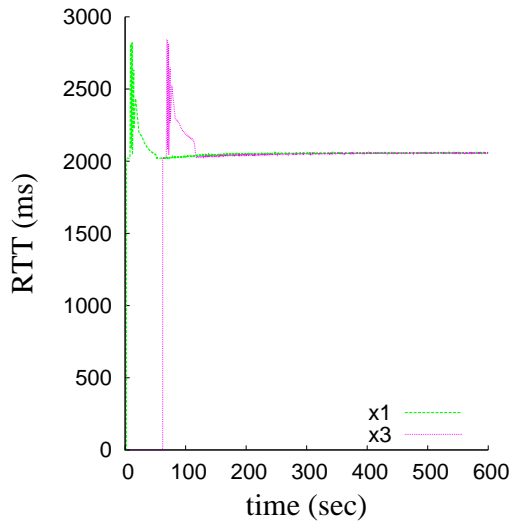

(c) iXCP long flow's RTT with ACK priority queue and estimation-control interval equal to max RTT

Fig. 36. iXCP outperforms JetMax in a highly dynamic environment with the arrivals and departures of web-like traffic.

aims to provide max-min fairness. Similar to our scheme, a Jetmax packet header includes a bottleneck identifier field, but its control algorithm is rate-based, completely different from XCP, which is a window-based protocol. Charny et al. [18] also proposes a rate-based algorithm to realize maxmin flow rate allocation, using a different control algorithm and feedback scheme. However, their approach requires perflow state at routers. In contrast, both XCP and $\mathrm{XXCP}$ are stateless.

Other work has focused on the implementation of XCP [19] and improvements of XCP in other areas. Zhang et al. [20] extensively studied the implementation and deployment challenges of XCP. Hsiao et al. [21] extended XCP to support streaming layered video. Kapoor et al. [22] proposes to combine XCP with a Performance Enhancement Proxy (PEP) to provide fast satellite access. Yang et al. [23] proposed an improvement to shorten XCP's response time for new flows to acquire their bandwidth. Zhang et al. [24] presented a control theoretical model that considers capacity estimation errors. XCP-r [25] proposes to calculate the congestion window size at the receiver side to cope with ACK losses.

\section{CONClusions And Future Work}

$\mathrm{XCP}[8]$ is a new and promising protocol that outperforms TCP in terms of efficiency, stability, queue size, and convergence speed. However, Low et al. [10] discovered a weakness of XCP. In some multi-bottleneck environments, XCP may only utilize as low as $80 \%$ of bottleneck bandwidth.
This paper proposes an improved XCP (iXCP) that solves the link under-utilization problem of XCP. We use extensive simulations as well as a theoretical analysis to show that $\mathrm{XCP}$ is able to efficiently utilize bottleneck bandwidth and is maxmin fair in steady state. iXCP also preserves other features of $\mathrm{XCP}$, including small queue size, near-zero packet drops, and fast convergence.

\section{APPENDIX \\ TheORETICAL MODEL OF IXCP}

In this section, we build on Low's theoretical model [10] to prove that iXCP achieves full link utilization and max-min fairness (Theorem 1 in Section III). As iXCP differs from $\mathrm{XCP}$ in its shuffling operation, we only show the derivation for feedback computed from the shuffling operation in detail, and use Low's result for the spare bandwidth analysis.

The high-level idea of our analysis is as follows. We first convert the per-packet feedback computed by iXCP's control algorithm to a per-flow feedback, and then derive a flow's rate at an equilibrium from the feedback, using the condition that in an equilibrium, the feedback for window adjustment is zero. We then show that the equilibrium rate is the max-min rate. Our analysis assumes that a flow has a fixed packet size.

We first define the following notations:

- $L$ : the set of links in the network.

- $N$ : the number of flows in the network.

- $i$ : the index of a flow.

- $l$ : the index of a link.

- $k$ : the index of a packet. 
- $R$ : the $L \times N$ routing matrix. $R_{l i}=1$ if flow $i$ uses link $l$, and 0 otherwise.

- $L(i)$ : the set of links in the path of each flow $i . L(i)=$ $\left\{l \mid R_{l i}=1\right\}$.

- $I(l)$ : the set of flows that cross the link $l: I(l)=\left\{i \mid R_{l i}=\right.$ $1\}$.

- $I_{1}(l)$ : the set of flows that cross the link $l$ and whose bottleneckId is the same as link $l$ 's identifier.

For each flow $i$, we define the following variables:

- $w_{i}(t)$ : the congestion window size at time $t$ (in packets).

- $T_{i}(t)$ : the round-trip time (RTT) at time $t$.

- $x_{i}(t)$ : the flow $i$ 's sending rate in unit of packets per second, and $x_{i}(t)=w_{i}(t) / T_{i}(t)$.

As each packet also carries a window size and a RTT in its congestion header, we use $k$ as the index for a packet to indicate the value carried in a packet. For instance, $w_{i, k}(t)$ is the window size carried by packet $k$ that belongs to flow $i$.

For each link $l$, we define the following variables:

- $c_{l}$ : the capacity (in packets per second).

- $b_{l}(t)$ : the queue size at time $t$ (in packets).

- $y_{l}(t)$ : the aggregate input traffic rate at time $t$, and $y_{l}(t)=$ $\sum_{i} R_{l i} x_{i}(t)$.

- $y_{l 0}(t)$ : the aggregate rate for flows crossing link $l$ but not bottlenecked at link $l$ at time $t$.

- $N_{l}$ : the number of flows crossing link $l . N_{l}=|I(l)|$.

- $N_{l 0}$ : the number of flows crossing link $l$ but not bottlenecked at link $l . N_{l 0}=|I(l)|-\left|I_{1}(l)\right|$

- $\phi_{l}(t)$ : the spare bandwidth computed by iXCP's control algorithm.

- $h_{l}(t)$ : the shuffled bandwidth computed by iXCP's control algorithm.

- $K_{l}(t)$ : the number of packets from flows that are bottlenecked at link $l$ during the control interval that proceeds time $t$.

- $P_{l, i}(t)$ : the total number of packets from flow $i$ during the control interval that proceeds time $t$.

- $r_{l}$ : the equilibrium rate allocated to a flow at the bottleneck link $l$.

Next, we define the control parameters of iXCP and the feedback computed by iXCP. The control parameters are the same as defined in XCP [8].

- $\alpha, \beta$ : the damping coefficients used to compute the spare bandwidth.

- $\gamma$ : the coefficient used to compute the shuffled bandwidth.

- $d$ : the control interval in unit of seconds.

- $p$ : the per-packet positive feedback. The variable $p$ may have four subscripts: 1) $l$ : the index of the link this positive feedback is computed from; 2) $i$ : the index of the flow a packet belongs to; 3) $k$ : the index of the packet for which the feedback is computed; 4) $\phi$ or $h$ : indicating whether the feedback is computed from the spare bandwidth or the shuffled bandwidth.

- $n$ : the per-packet negative feedback. The subscript definitions for $n$ are the same as those for $p$.

- $H$ : the overall per-packet feedback, and $H=p-n$. The subscripts for $H$ are the same as those for $p$ and $n$.
Theorem A. iXCP achieves max-min fair bandwidth allocation in steady state. That is, with $i X C P$, every flow has a bottleneck. And a link is called bottleneck for a flow if and only if its sending rate is larger than or equal to all other flows passing through that link.

Proof: The spare bandwidth and shuffled bandwidth at link $l$ are defined by the following equations:

$$
\begin{aligned}
& \phi_{l}(t)=\alpha d\left(c_{l}-y_{l}(t)\right)-\beta b_{l}(t) \\
& h_{l}(t)=\max \left\{0, \gamma\left(y_{l}(t)-y_{l 0}(t)\right)-\left|\phi_{l}(t)\right|\right\}
\end{aligned}
$$

From iXCP's control algorithm, we can represent the perpacket positive feedback and negative feedback computed from the shuffled bandwidth on packet $k$ as follows:

$$
\begin{aligned}
p_{l, i, k, h}(t) & =h_{l}(t) \frac{T_{i, k}(t)}{d} \frac{T_{i, k}(t) / w_{i, k}(t)}{\sum_{u \in I_{1}(l)} \sum_{v \in P_{l, u}(t)} T_{u, v}(t) / w_{u, v}(t)}(3) \\
n_{l, i, k, h}(t) & =h_{l}(t) \frac{T_{i, k}(t)}{d} \frac{1}{K_{l}(t)}
\end{aligned}
$$

We note that the feedbacks computed for packets from the same flow are the same. We then convert the per-packet feedback into a per-flow feedback. Let the flow rate computed from a packet be $x_{i, k}(t)=w_{i, k}(t) / T_{i, k}(t)$ and replace it in (3), we obtain:

$$
p_{l, i, k, h}(t)=h_{l}(t) \frac{T_{i, k}(t)}{d} \frac{1 / x_{i, k}(t)}{\sum_{u \in I_{1}(l)} \sum_{v \in P_{l, u}(t)} 1 / x_{u, v}(t)}(5)
$$

The number of packets from a flow received within a control interval equals to the rate of the flow times the length of the control interval: $P_{l, i}(t)=R_{l i} x_{i}(t) d$, and $x_{i, k}=x_{i}$. We have:

$$
\begin{aligned}
\sum_{u \in I_{1}(l)} \sum_{v \in P_{l, u}(t)} 1 / x_{u, v}(t) & =\sum_{u \in I_{1}(l)} R_{l u} x_{u}(t) d \frac{1}{x_{u}(t)} \\
& =\left(N_{l}-N_{l 0}\right) d
\end{aligned}
$$

By substituting (6) in (5), we obtain:

$$
p_{l, i, k, h}(t)=\frac{T_{i, k}(t)}{d^{2}} \frac{h_{l}(t)}{\left(N_{l}-N_{l 0}\right) x_{i, k}}
$$

The number of packets from flows that are bottlenecked at the link $l$ equals to the aggregate rates from those flows times the control interval:

$$
K_{l}(t)=\left(y_{l}(t)-y_{l 0}(t)\right) d
$$

By plugging (7) into (4), we obtain the negative feedback:

$$
n_{l, i, k, h}(t)=\frac{T_{i, k}(t)}{d^{2}} \frac{h_{l}(t)}{y_{l}(t)-y_{l 0}(t)}
$$

Therefore, the net feedback from the shuffled bandwidth is:

$$
H_{l, i, k, h}(t)=\frac{T_{i, k}(t)}{d^{2}}\left(\frac{h_{l}}{\left(N_{l}-N_{l 0}\right) x_{i, k}(t)}-\frac{h_{l}}{y_{l}(t)-y_{l 0}(t)}\right)
$$

From Low's model [10], the feedback from the spare bandwidth is computed as:

$$
H_{l, i, k, \phi}(t)=\frac{T_{i, k}(t)}{d^{2}}\left(\frac{\phi_{l}^{+}}{N_{l} x_{i, k}(t)}-\frac{\phi_{l}^{-}}{y_{l}(t)}\right)
$$

where $\phi_{l}^{+}(t)=\max \left(\phi_{l}(t), 0\right)$ and $\phi_{l}^{-}(t)=\max \left(-\phi_{l}(t), 0\right)$.

Putting it all together, the feedback for packet $k$ from both the spare bandwidth and the shuffled bandwidth is:

$$
H_{l, i, k}(t)=H_{l, i, k, \phi}(t)+H_{l, i, k, h}(t)
$$


Since flow $i$ receives $x_{i}(t)$ feedback packets per unit time, its window evolves according to the minimum feedback from its packets:

$$
\Delta w_{i}(t)=x_{i}(t) \min _{l \in L(i)} H_{l, i, k}(t)
$$

In an equilibrium, a flow's window will stop changing [10], $\Delta w_{i}(t)=0$, which leads to $\min _{l \in L(i)} H_{l, i, k}(t)=0$. Note that the flow's rate and feedbacks will not change over time in an equilibrium. So we can drop the time variable $t$, and the packet index $k$. Replacing the terms in $H_{l, i, k}$, we have:

$$
\min _{l \in L(i)}\left(\frac{\phi_{l}^{+}}{N_{l} x_{i}}-\frac{\phi_{l}^{-}}{y_{l}}+\frac{h_{l}}{\left(N_{l}-N_{l 0}\right) x_{i}}-\frac{h_{l}}{y_{l}-y_{l 0}}\right)=0
$$

We obtain this condition for all flows $i$ at all links the flow crosses, i.e., $l \in L(i)$. Therefore, we have:

$$
x_{i} \leq \frac{y_{l}\left(y_{l}-y_{l 0}\right)\left(\phi_{l}^{+}\left(N_{l}-N_{l 0}\right)+h_{l} N_{l}\right)}{N_{l}\left(N_{l}-N_{l 0}\right)\left(\phi_{l}^{-}\left(y_{l}-y_{l 0}\right)+h_{l} y_{l}\right)}
$$

The equality holds for flows that are bottlenecked at link $l$. Note that the right side of (9) does not depend on the flow index $i$. So all flows that are bottlenecked at a link $l$ must have the same rate $r_{l}$. That is:

$$
x_{i}=r_{l}, \forall i \in I_{1}(l)
$$

On the other hand, from the definitions of $y_{l}, y_{l 0}, N_{l}$, and $N_{l 0}$, we can compute the common rate $r_{l}$ as

$$
r_{l}=\frac{y_{l}-y_{l 0}}{N_{l}-N_{l 0}}
$$

Moreover, only one of $\phi_{l}^{+}$and $\phi_{l}^{-}$can be non-zero. Here we ssume $\phi_{l}^{+}$is non-zero (similar proof can be shown when $\phi_{l}^{-}$is non-zero). Thus, $\phi_{l}^{+}=\phi_{l}$. We have:

$$
\frac{y_{l}-y_{l 0}}{N_{l}-N_{l 0}} \frac{\phi_{l}\left(N_{l}-N_{l 0}\right)+h_{l} N_{l}}{h_{l} N_{l}}=\frac{y_{l}-y_{l 0}}{N_{l}-N_{l 0}}
$$

The above equality leads to $\phi_{l}=0$, which shows that the link utilization is $100 \%$. For all flows $x_{m}$, there , in equilibrium, exists a link $l$ such that $H_{l, m, k}(t)=$ $\min _{l \in L(m)} H_{l, m, k}(t)=0$. And for all $x_{n} \in I(l), H_{l, n, k}(t) \geq$ 0 holds. So we have $x_{n} \leq x_{m}$ from (9). What's more, $\phi_{l}=0$ leads to $y_{l}(t)=c_{l}$. Therefore link $l$ is the bottleneck for $x_{m}$. Since every flow has a bottleneck, it follows from [11] that the equilibrium rate is the max-min fair allocation.

Note that the above theorem is correct only when each flow identifies its bottleneck link correctly. That is each flow's bottleneckId is equal to link who gives zero feedback in the equilibrium. In the next, we show that a flow's bottleneckId will converge to its correct bottleneck. We first give the following lemmas:

Lemma A. With arbitrary fixed bottleneckId setting for each flow, for any flow $i$ and link $l$, if $x_{i}(t) \leq x_{j}(t) \forall j \in I_{1}(l)$, then the feedback for flow $i, H_{l, i, k}(t) \geq 0$. The equality holds iff $x_{i}(t)=x_{j}(t) \forall j \in I_{1}(l)$ and $H_{l, i, k, \phi}(t)=0$.

Proof: We assume each link is never overshooted and therefore we have $H_{l, i, k, \phi}(t) \geq 0$. Moreover, $H_{l, i, k, h}(t)=\frac{h_{l}(t) * T_{i}(t)}{d}\left(\frac{T_{i}(t) / w_{i}(t)}{\sum_{j \in I_{1}(l)} T_{j}(t) / W_{j}(t)}-\frac{S_{i}}{\sum_{j \in I_{1}(l)} S_{j}}\right)$ $=\frac{h_{l}(t) * T_{i}(t)}{d}\left(\frac{S_{i} / x_{i}(t)}{\sum_{j \in I_{1}(l)} S_{j} / x_{j}(t)}-\frac{S_{i}}{\sum_{j \in I_{1}(l)} S_{j}}\right) \geq 0$ because $x_{i}(t) \leq x_{j}(t) \forall j \in I_{1}(l)$. Therefore $H_{l, i, k}(t)=H_{l, i, k, \phi}(t)+$ $H_{l, i, k, h}(t) \geq 0$. And it is obvious that the equality holds iff $x_{i}(t)=x_{j}(t) \forall j \in I_{1}(l)$ and $H_{l, i, k, \phi}(t)=0$.

Lemma B. With arbitrary fixed bottleneckId setting for each flow, for any flow $i$ and link $l$, if $x_{i}(t)>x_{j}(t) \forall j \in I_{1}(l)$ and $H_{l, i, k, \phi}(t)=0$, then the feedback for flow $i, H_{l, i, k}(t)<0$.

$$
\text { Proof: } H_{l, i, k, h}(t)=\frac{h_{l}(t) * T_{i}(t)}{d}\left(\frac{T_{i}(t) / w_{i}(t)}{\sum_{j \in I_{1}(l)} T_{j}(t) / W_{j}(t)}-\right.
$$
$\left.\frac{S_{i}}{\sum_{j \in I_{1}(l)} S_{j}}\right)=\frac{h_{l}(t) * T_{i}(t)}{d}\left(\frac{S_{i} / x_{i}(t)}{\sum_{j \in I_{1}(l)} S_{j} / x_{j}(t)}-\frac{S_{i}}{\sum_{j \in I_{1}(l)} S_{j}}\right)<0$ because $x_{i}(t) \geq x_{j}(t) \forall j \in I_{1}(l)$. Therefore $H_{l, i, k}(t)=$ $H_{l, i, k, \phi}(t)+H_{l, i, k, h}(t)<0$.

Theorem B. All flows' bottleneckIds converge to their correct bottleneck.

Proof: In order to make the analysis tractable, we make the following assumptions. We assume that all flows switch their bottleneck synchronously and that all flows have the same RTT and start transmission at the same time. Our proof proceeds in discrete steps. A step starts when each host starts the back-to-back transmission of all packets falling within the congestion window. Each host does not change its window size or bottleneckId until the next step begins. Once all endhosts receive all the ACKs for all the packets sent out in this step, this step ends. Then each endhost adjusts the window size in terms of the aggregated feedback of these ACKs and updates its bottleneckId according to the nextBottleneckId in the last ACK, which marks the beginning of the next step. These assumptions, despite being simplistic, are also used in the related works [8], [26], [27].

We use $r(n)$ to denote the smallest flow rate in the system at step $n$. We refer to a flow's bottleneckId link as the link whose identifier matches the flow's bottleneckId field. We also refer to a flow's common bottleneckId flows as all of the flows whose bottleneckId is the same as that flow including itself.

The proof sketch is as follows. We prove the smallest flows in the system will enter a stable state for them within a limited number of steps. Then we remove the smallest flows from the system and repeat the argument again until the system is empty. The detailed proof is presented below.

We first show that, at any step $n$, as long as there are flows whose rate is larger than $r(n)$ passing through the smallest flows' bottleneckId link, then there is a $t<\infty, t \in Z^{+}$such that $r(n+t)>r(n)$. In other words, the smallest flow rate of the system increases within a limited number of steps. To see this, we first note that $r(n)$ cannot decrease. This is because each flow is trying to reach the average flow rate of its common bottleneckId flows and the converging process is monotonic. So any flow rate in this step cannot be smaller than the smallest flow rate in the previous step. We also need to note that flows with a rate larger than $r(n)$ at step $n$ must end up with a rate still larger than $r(n)$ at step $n+1$. So if $r(n)=r(n+1)$, it is only possible that the smallest flows at step $n+1$ are also the smallest flows at step $n$. Next suppose $r(n)$ remains the same while there are larger flows passing through the smallest flows' bottleneckId link. Then there are two cases. Case 1: the larger flows' bottleneckId is the same as the smallest flows'. Then the smallest flows will receive positive feedback as per Lemma A 
and increase their rate, contradictory to the supposition. Case 2: the larger flows' bottleneckId is different from the smallest flows'. Then spare bandwidth for smallest flows' bottleneckId link must be zero; otherwise smallest flows will get positive feedback. In this case, the larger flows will keep receiving negative feedback as per Lemma B and, sooner or later, it will switch its bottleneckId to this link. Then the contradiction follows Case 1.

We next show that, within a limited number of steps, system will go into a state such that smallest flows' bottleneckId link only contains smallest flows. We also prove this by contradiction. Suppose the system never goes into this state, then according to last paragraph's conclusion, the smallest flow rate in the system will keep increasing. Since the smallest system rate is bounded by the largest capacity of all links in the system, it renders contradiction.

Finally we show, in the above state, the smallest flows' bottleneckIds have converged to their correct bottleneck. First, the smallest flows in this state will not be able to change its rate anymore. Moreover, these smallest flows will receive positive feedback as per Lemma A from all links except bottleneckId links, which is the correct bottleneck for the smallest flows according to the definition of bottleneck [11]. Therefore the bottleneckId for the smallest flows in this state is correct and stable. We call the above state the stable state for smallest flows.

When the system reaches stable state for its smallest flows. We simply remove these smallest flows from the system and the remaining system will end up with a new stable state for some new smallest flows in a limited number of steps.

We repeate the above argument again and again until there are no more flows in the system. Then, within a limited number of steps, the system will be empty, that is, all flows' bottleneckIds converge to a correct bottleneck within a limited number of steps.

\section{REFERENCES}

[1] S. Low, F. Paganini, J. Wang, S. Adlakha, and J. Doyle, "Dynamics of TCP/RED and a Scalable Control," in Proc. of Infocom, 2002.

[2] K. Zhou, K. Yeung, and V. Li, "P-XCP: A Transport Layer Protocol for Satellite IP Networks," in Proc. of GLOBECOM, 2004, pp. 2707-2711.

[3] Y. Zhang, D. Leonard, and D. Loguinov, "JetMax: Scalable Maxmin Congestion Control for High-Speed Heterogeneous Networks," in Proc. of Infocom, 2006.

[4] C. Jin, D. Wei, and S. Low, "Fast TCP: Motivation, Architecture, Algorithms, Performance," in Proc. of Infocom, March 2004.

[5] S. Floyd, "HighSpeed TCP for Large Congestion Windows," in IETF RFC 3649, Dec. 2003.

[6] T. Kelly, "Scalable TCP: Improving Performance in HighSpeed Wide Area Networks," in 1st International Workshop on PFLDN, Feb. 2003.

[7] L. Xu, K. Harfoush, and I. Rhee, "Binary increase congestion control for fast long-distance networks," in Proc. of Infocom, Mar. 2004.

[8] D. Katabi, M. Handley, and C. Rohrs, "Congestion Control for High Bandwidth-Delay Product Networks," in Proc. of Sigcomm, 2002.

[9] D. Clark, K. Sollins, J. Wroclawski, D. Katabi, J. Kulik, X. Yang, R. Braden, T. Faber, A. Falk, V. Pingali, M. Handley, and N. Chiappa, "New Arch: Future Generation Internet Architecture," USC ISI, Tech. Rep., 2003. [Online]. Available: http://www.isi.edu/newarch/

[10] S. Low, L. Andrew, and B. Wydrowski, "Understanding XCP: Equilibrium and Fairness," in Proc. of Infocom, 2005, pp. 1025-1036.

[11] D. Bertsekas and R. Gallager, "Data Networks," in Prentics-Hall Inc., 2nd ed., 1992.
[12] iXCP@UCI. [Online]. Available: http://www.ics.uci.edu/ yanbinl/ixcp/ ixcp.html

[13] "Caida report," February 2000. [Online]. Available: http://www.caida. org/analysis/AIX/plen_hist/

[14] The network simulation: ns2. [Online]. Available: http://www.isi.edu/ nsnam/ns

[15] M. Crovella and A. Bestavros, "Self-Similarity in World Wide Web Traffic: Evidence and Possible Causes," in Proc. of IEEE/ACM Trans. Networking, 1997.

[16] L. L. H. Andrew, B. P. Wydrowski, and S. H. Low, "An example of instability in xcp," 2005. [Online]. Available: http: //www.cs.caltech.edu/ lachlan/abstract/xcpInstability.pdf

[17] S. Jain, Y. Zhang, and D. Loguinov, "Towards Experimental Evaluation of Explicit Congestion Control," in Proc. of IWQoS, 2008.

[18] A. Charny, D. Clark, and R. Jain, "Congestion Control With Explicit Rate Indication," in Proc. of ICC, 1995.

[19] A. Falk and D. Katabi, "Specification for the Explicit Control Protocol (XCP)," 2005. [Online]. Available: http://www.isi.edu/isi-xcp/ docs/draft-falk-xcp-spec-01.txt

[20] Y. Zhang and T. Henderson, "An Implementation and Experimental Study of the eXplicit Control Protocol (XCP)," in Proc. of Infocom, 2005, pp. 1037-1048.

[21] H. Hsiao and J. Hwang, "A Max-Min Fairness Congestion Control for Streaming Layered Video," in Proc. of ICASSP, 2004, pp. V981- V984.

[22] A. Kapoor, A. Falk, T. Faber, and Y. Pryadkin, "Achieving faster access to satellite link bandwidth," in Proc. of Infocom, 2005, pp. 2870-2875.

[23] Y. Yang, C. Chan, P. Wang, and Y. Chen, "Performance Enhancement of XCP for High Bandwidth-Delay Product Networks," in Proc. of ICACT, 2005, pp. 456-461.

[24] Y. Zhang and M. Ahmed, "A Control Theoretic Analysis of XCP," in Proc. of Infocom, 2005, pp. 2831-2835.

[25] D. Lopez-Pacheco and C. Pham, "Robust Transport Protocol for Dynamic High-Speed Networks: Enhancing the XCP Approach," in Proc. of IEEE MICC-ICON, Nov. 2005.

[26] S. Kandula, D. Katabi, B. Davie, and A. Charny, "Walking the tightrope: responsive yet stable traffic engineering," in Proc. of Sigcomm, 2005.

[27] J. Padhye, V. Firoiu, D. Towsley, and J. Kurose, "Modeling tcp throughput: A simple model and its empirical validation," in Proc. of Sigcomm, 1998.

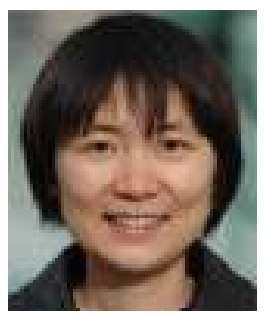

Xiaowei Yang received the B.E. degree in electronic engineering from Tsinghua University, Beijing, China, in 1996 and a Ph.D. degree in computer science from Massachusetts Institute of Technology in 2004. Currently, she is an assistant professor of Computer Science at the University of California, Irvine. Her research interests include congestion control, quality of service, Internet routing architecture, and network security.

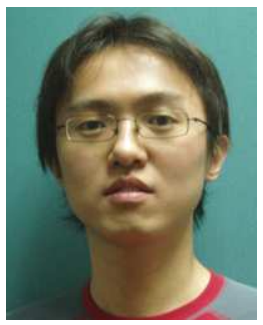

Yanbin Lu received the B.S. degree in computer science from Beijing University of Posts and Telecommunications, Beijing, China, in 2004 and the M.S. degree in computer science from Graduate University of Chinese Academy of Sciences, Beijing, China, in 2007 . He is currently working towards the $\mathrm{Ph} . \mathrm{D}$. degree in computer science at the University of California, Irvine, CA, USA. His research interests include resource allocation, routing technique and network security. 


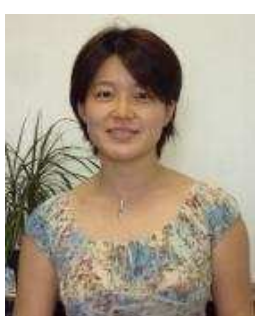

Lei Zan received the B.S. degree in electronic engineering from Peking University, Beijing, China, in 1998; M.S. degree in electronic engineering from Hong Kong University of Science and Technology, Hongkong, China, in 2001; M.S. degree in computer science from University of California, Irvine, CA, USA in 2007. 